\title{
Mukai's program for curves on a K3 surface
}

\author{
E. Arbarello, A. Bruno and E. Sernesi
}

\begin{abstract}
Let $C$ be a general element in the locus of curves in $M_{g}$ lying on some K3 surface, where $g$ is congruent to $3 \bmod 4$ and greater than or equal to 15. Following Mukai's ideas, we show how to reconstruct the K3 surface as a Fourier-Mukai transform of a Brill-Noether locus of rank two vector bundles on $C$.
\end{abstract}

\section{Introduction}

Let $\mathcal{K}_{g}$ be the moduli stack of pairs $(S, H)$ where $S$ is a $\mathrm{K} 3$ surface and $H$ is a very ample line bundle on $S$ such that $H^{2}=2 g-2$. Let $\mathcal{P}_{g}$ be the stack of pairs $(S, C)$ with $(S, H) \in \mathcal{K}_{g}$ and $C \in|H|$ smooth and irreducible. Finally, let $\mathcal{M}_{g}$ be the moduli stack of smooth curves of genus $g$. The stacks $\mathcal{K}_{g}, \mathcal{P}_{g}, \mathcal{M}_{g}$ are smooth Deligne-Mumford stacks of dimensions $19,19+g, 3 g-3$ respectively. We have natural morphisms

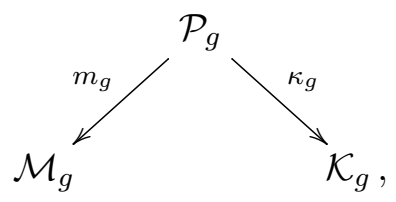

where $\kappa_{g}$ realizes $\mathcal{P}_{g}$ as an open subset of a $\mathbb{P}^{g}$-fibration. In [CLM93, Theorem 5], the authors prove that for $g=11$ and $g \geqslant 13$, the morphism $m_{g}$ is a birational map onto its image using properties of the Gauss map for the canonical divisor (also known as Wahl map). Related references are [Wah97], [Wah02].

On the last page of [Muk01], Mukai laid out a beautiful program to actually reconstruct a K3 surface from a curve lying on it, thus giving a rational inverse of $m_{g}$ whenever the genus $g$ is congruent to $3 \mathrm{mod} 4$ and greater than or equal to 11 . This program was successfully carried out by him for the case $g=11$ in [Muk96].

In our work we take Mukai's paper [Muk96] as a blueprint and generalize it to all genera which are congruent to $3 \bmod 4$ and greater than or equal to 11.

Let $(S, C)$ be a general point in $\mathcal{P}_{g}$, where $g=2 s+1$ with $s \geqslant 5$ odd. Mukai's strategy to reconstruct the surface $S$ from the curve $C$ is as follows. Consider the Brill-Noether locus $M_{C}\left(2, K_{C}, s\right)$, which is the moduli space of semistable rank-two vector bundles on $C$ having canonical determinant and possessing at least $s+2$ linearly independent sections. Then $M_{C}\left(2, K_{C}, s\right)$ is a K3 surface and the surface $S$ can be obtained as an appropriate Fourier-Mukai transform of it.

Received 16 September 2013, accepted in final form 27 April 2014.

2010 Mathematics Subject Classification 14J28 (primary), 14D20 (secondary)

Keywords: K3 surface, vector bundle, stability

This journal is (C) Foundation Compositio Mathematica 2014. This article is distributed with Open Access under the terms of the Creative Commons Attribution Non-Commercial License, which permits non-commercial reuse, distribution, and reproduction in any medium, provided that the original work is properly cited. For commercial re-use, please contact the Foundation Compositio Mathematica. 


\section{MUKAI PROGRAM}

When $g=11$ the proof consists of three main steps. One first considers pairs $\left(S^{\prime}, C^{\prime}\right)$ where $S^{\prime}$ is a K3 surface of a special type, and proves with ad-hoc constructions that $M_{C^{\prime}}\left(2, K_{C^{\prime}}, s\right)$ is isomorphic to $S^{\prime}$ by showing that both are isomorphic to a moduli space $M_{v}\left(S^{\prime}\right)$ of vector bundles on $S^{\prime}$. The second step consists in deforming $\left(S^{\prime}, C^{\prime}\right)$ to a general pair $(S, C)$ : since $M_{C}\left(2, K_{C}, 5\right)$ has expected dimension equal to two it is a flat deformation of $M_{C^{\prime}}\left(2, K_{C^{\prime}}, s\right)$, thus it is again a K3 surface. Finally, one shows the existence of an appropriate polarization $h$ on $M_{C}\left(2, K_{C}, 5\right)$ which induces an isomorphism between $S$ and the Fourier-Mukai transform of $M_{C}\left(2, K_{C}, 5\right)$ with respect to $h$.

The first difficulty in trying to extend this proof is that when $g=2 s+1$ with $s \geqslant 6$, the expected dimension of $M_{C}\left(2, K_{C}, s\right)$ is zero for $s=6$ and negative for $s \geqslant 7$, so that it is not even clear that $M_{C}\left(2, K_{C}, s\right)$ is non-empty when $s \geqslant 7$. However, in her paper [Voi92], Voisin associates a rank-two vector bundle $E_{L}$ to each base-point-free pencil $|L|$ on $C$ of degree $s+2$. Each of these bundles is exhibited as an extension

$$
0 \longrightarrow K_{C} L^{-1} \longrightarrow E_{L} \longrightarrow L \longrightarrow 0
$$

and one can prove that the Voisin bundles $E_{L}$ are stable (see, for instance, Lemma 2.5, Proposition 3.1 and Remark 5.11) and that, as $L$ varies in $W_{s+2}^{1}(C)$, they describe a one-dimensional locus in $M_{C}\left(2, K_{C}, s\right)$.

Consider on the K3 surface $S$ the Mukai vector $v=(2,[C], s)$ and denote by $M_{v}(S)$ the moduli space of $[C]$-stable, rank-two vector bundles $E$ on $S$ with $c_{1}(E)=[C]$ and $\chi(S, E)=s+2$. For a general K3 surface, $M_{v}(S)$ is again a smooth K3 surface. One of our main results is the following.

Theorem 6.1. For a general point $(C, S) \in \mathcal{P}_{g}$, where $g=2 s+1$ with $s \geqslant 5$, there is a unique irreducible component $V_{C}\left(2, K_{C}, s\right)$ of $M_{C}\left(2, K_{C}, s\right)$ containing the Voisin bundles $E_{L}$. By sending $\mathcal{E}$ to $\mathcal{E}_{\mid C}$ one obtains a well-defined isomorphism

$$
\sigma: M_{v}(S) \longrightarrow V_{C}\left(2, K_{C}, s\right)_{\text {red }} .
$$

In particular, $V_{C}\left(2, K_{C}, s\right)_{\text {red }}$ is a smooth $\mathrm{K} 3$ surface.

Note that we only assumed $g$ to be odd in Theorem 6.1. Let now $M_{C}\left(2, K_{C}\right)$ be the moduli space of rank-two vector bundles on $C$ with determinant equal to $K_{C}$. Write, for simplicity, $T=V_{C}\left(2, K_{C}, s\right)_{\text {red }} \subset M_{C}\left(2, K_{C}\right)$. Following Mukai's program, let $\mathcal{U}$ be a universal bundle on $C \times T$, let $\pi_{C}$ and $\pi_{T}$ be the natural projections from $C \times T$ to $C$ and $T$, respectively, and consider the determinant of the cohomology

$$
h_{\text {det }}=\left(\operatorname{det} R^{1} \pi_{T *} \mathcal{U}\right) \otimes\left(\operatorname{det} \pi_{T *} \mathcal{U}\right)^{-1} .
$$

For $s$ odd, (that is, $g \equiv 3 \bmod 4$ ), we prove that $h_{\text {det }}$ is a genus $g$ polarization on $T$ and that $\mathcal{U}$ can be chosen in such a way that the map

$$
C \longrightarrow \widehat{T}=M_{\widehat{v}}(T), \quad x \longmapsto \mathcal{U}_{\mid\{x\} \times T}
$$

is an embedding. We then have the following theorem (see also the more detailed statement in $\S 7)$.

Theorem 7.1. Let $(C, S)$ be a general point of $\mathcal{P}_{g}$, where $g=2 s+1$ with $s \geqslant 5$ odd. Let $T=$ $V_{C}\left(2, K_{C}, s\right)_{\text {red }}$. Consider the Mukai vector $\widehat{v}=\left(2, h_{d e t}, s\right)$. Then any $\mathrm{K} 3$ surface containing $C$ is isomorphic to $\widehat{T}=M_{\widehat{v}}(T)$.

In proving both Theorem 6.1 and Theorem 7.1, the basic tool consists in degenerating the 


\section{E. Arbarello, A. Bruno and E. Sernesi}

surface $S$ to a rather special K3 surface where both the geometry of the moduli space $M_{v}(S)$ and the properties of the morphism $\sigma: M_{v}(S) \rightarrow V_{C}\left(2, K_{C}, s\right)_{r e d}$ are made transparent by virtue of an explicit isomorphism $S \cong M_{v}(S)$ (see Proposition 5.7).

The special K3 surfaces we consider are the direct generalizations of those considered by Mukai in his analysis of the genus eleven case. Namely, we consider a K3 surface $S$ such that

$$
\operatorname{Pic}(S)=\mathbb{Z} \cdot[A] \oplus \mathbb{Z} \cdot[B]
$$

with

$$
|C|=|A+B| \quad \text { and } \quad g(A)=s, g(B)=1 .
$$

This means that on $C$, the elliptic pencil $|B|$ cuts out a $g_{s+1}^{1}$ which we call $\xi$, while the linear system $|A|$ cuts out the residual series, a $g_{3 s-1}^{s}$ which we call $\eta$. An isomorphism

$$
\rho: S \longrightarrow M_{v}(S)
$$

is obtained by assigning to each $x \in S$ the vector bundle $\mathcal{E}_{x}$ defined as the unique extension

$$
0 \longrightarrow \mathcal{O}_{S}(B) \longrightarrow \mathcal{E}_{x} \longrightarrow I_{x}(A) \longrightarrow 0 \text {. }
$$

The isomorphism $\rho$ makes $S$ self-dual, from the Fourier-Mukai point of view. Such self-duality is the key to proving Theorem 6.1 for pairs $(C, S)$ satisfying (1.3) and (1.4). Moreover, in this case $S \cong M_{C}\left(2, K_{C}, s\right)=V_{C}\left(2, K_{C}, s\right)$ (Theorem 5.1). The geometry of the special surface $S$ is quite different from the $g=11$ case and requires a number of new auxiliary results that are proved in $\S 4$. Moreover, the negativity of the expected dimension of $M_{C}\left(2, K_{C}, s\right)$ is the reason for some lengthening in the proof of Theorem 5.1.

The embedding of $S$ in $\mathbb{P}^{s}$ via the linear series $|A|$ also plays a fundamental role. Denote by $T$ and $\Gamma$ the images of $S$ and $C$, respectively, via this embedding. Then the quadratic hull of $T$ coincides with the quadratic hull of $\Gamma$ and, as such, it classifies extensions on $C$ :

$$
0 \longrightarrow \xi \longrightarrow E \longrightarrow \eta \longrightarrow 0 \text {. }
$$

The rank-two vector bundles $E$, or better their stable models, obtained in this way parametrize $M_{C}\left(2, K_{C}, s\right)$ giving, via (1.5), a geometrical interpretation of the isomorphism (1.2).

In the general case, Theorem 6.1 is proved by a variational argument similar to Mukai's, with the added difficulty coming from the negativity of the expected dimension of the Brill-Noether locus. We consider a family of pairs $(S, C)$ with a special fibre satisfying (1.3) and containing a general pair with Picard rank one among its fibres. By applying some deformation theory arguments we are able to control the behaviour of the map (1.5) on the general fibre, overcoming the fact that $M_{C}\left(2, K_{C}, s\right)$ has negative expected dimension.

\section{Moduli of sheaves on a K3 surface}

Let $(S, C)$ be a pair consisting of a K3 surface $S$ and a non-singular curve $C \subset S$ of genus

$$
g(C)=g=2 s+1
$$

for some $s \geqslant 5$. We let $M_{v, C}(S)$ be the moduli space of $[C]$-semistable sheaves with Mukai vector $v$ on $S$ and polarization $[C]$. The Mukai vector of a sheaf $F$ is given by

$$
v(F)=\left(r(F), c_{1}(F), \chi(F)-r(F)\right),
$$




\section{MUKAI PROGRAM}

where $r(F)$ denotes the rank of $F$. From now on we consider the case in which the Mukai vector $v$ is given by

$$
v=(2,[C], s)
$$

and we will write $M_{v, C}(S)=M_{v}(S)$.

As already anticipated in the introduction, in this paper we will mostly consider the following two cases, to which we give a name.

- Rank-1 case: $\operatorname{Pic}(S)=\mathbb{Z} \cdot[C]$.

- Rank-2 case: $\operatorname{Pic}(S)=\mathbb{Z} \cdot[A] \oplus \mathbb{Z} \cdot[B]$ with $[C]=[A+B], A$ and $B$ non-singular connected and $g(A)=s, g(B)=1$. We then write

$$
\mathcal{O}(B)_{\mid C}=\xi, \quad \mathcal{O}(A)_{\mid C}=\eta,
$$

so that $\xi$ is a $g_{s+1}^{1}$ and $\eta$, the residual of $\xi$, is a $g_{3 s-1}^{s}$. For the existence of K3 surfaces of this type we refer to [Knu02, Theorem 1.1].

Lemma 2.1. In both the Rank-1 and the Rank-2 cases, the Mukai vector (2.1) is primitive and there are no walls relative to $v$.

Proof. In the Rank-1 case the lemma is obvious. Let us assume that we are in the Rank-2 case. The curve $C$ is clearly primitive and therefore $v$ is primitive as well. Let us show that there are no walls relative to $v$ in the ample cone of $S$ (see [Huy97, Definition 4.C.1]). Following the notation of [Huy97, Theorem 4.C.3], it suffices to prove that there is no element

$$
\lambda=2 c_{1}\left(F^{\prime}\right)-c_{1}(F),
$$

where $F$ is a $\mu_{C}$-semistable sheaf in $M_{v, C}(S)$ and $F^{\prime} \subset F$ is a rank-one subsheaf with $\mu_{C}\left(F^{\prime}\right)=$ $\mu_{C}(F)$, such that

$$
-\Delta \leqslant \lambda^{2} \leqslant 0
$$

where $\Delta=4 c_{2}(F)-c_{1}^{2}(F)=4(s+2)-4 s=8$. We may write $c_{1}\left(F^{\prime}\right)=h A+k B$. Now $(2.2)$ reads

$$
-8 \leqslant(2 h-1)^{2}(2 s-2)+2(2 k-1)(2 h-1)(s+1) \leqslant 0 .
$$

The equality $\mu_{C}\left(F^{\prime}\right)=\mu_{C}(F)$ gives $2 h(s-1)+k(s+1)=2 s$ and the inequalities above can be written as

$$
-4 \leqslant-s(2 h-1)^{2} \leqslant 0,
$$

which has no solutions for $s \geqslant 5$. So there are no walls.

Lemma 2.2. Assume that the Mukai vector (2.1) is primitive and that there are no walls relative to $v$. Then the moduli space $M_{v}(S)$, if not empty, is a smooth $K 3$ surface and all of its points represent locally free sheaves. In particular, this happens if we are in the Rank-1 or in the Rank-2 case.

Proof. Applying [Huy97, Theorem 4.C.3 and Lemma 1.2.13], we deduce that all sheaves in $M_{v}(S)$ are $[C]$-stable. Since $v$ is isotropic it follows that $M_{v}(S)$ is a smooth and irreducible projective surface ([Huy97, Theorem 6.1.8]) which is a K3 by [Muk84]. Since in this case [C]-stability is equivalent to $\mu$-stability, it follows from [Huy97, Remark 6.1.9, p. 145] that all the points of $M_{v}(S)$ represent locally free sheaves. The last assertion is a consequence of Lemma 2.1.

From the lemma it follows that, under its assumptions, $[C]$-(semi)stability is computed in 


\section{E. Arbarello, A. Bruno and E. Sernesi}

terms of the $C$-slope, which is defined by

$$
\mu_{C}(F)=\frac{c_{1}(F) \cdot C}{r(F)} .
$$

In particular, this happens if we are in the the Rank-1 or in the Rank-2 case. Let us recall the definition of Lazarsfeld-Mukai bundle.

Definition 2.3. Let $L$ be a globally generated pencil on $C \subset S$. The Lazarsfeld-Mukai bundle $\widetilde{E}_{L}$ is the dual of the rank-two vector bundle $\widetilde{F}_{L}$ defined by the exact sequence

$$
0 \longrightarrow \widetilde{F}_{L} \longrightarrow H^{0}(L) \otimes \mathcal{O}_{S} \stackrel{e v}{\longrightarrow} L \longrightarrow 0 \text {. }
$$

REMARK 2.4. Often, in the literature, the bundle $\widetilde{F}_{L}$ is denoted by the symbol $F_{C, L}$ and its dual bundle $\widetilde{E}_{L}$ is denoted by the symbol $E_{C, L}$.

For these bundles one easily computes the basic invariants:

$$
\begin{aligned}
r\left(\widetilde{E}_{L}\right) & =2, \quad c_{1}\left(\widetilde{E}_{L}\right)=[C], \quad c_{2}\left(\widetilde{E}_{L}\right)=\operatorname{deg} L, \\
h^{0}\left(\widetilde{F}_{L}\right) & =h^{0}\left(\widetilde{E}_{L}(-C)\right)=h^{2}\left(\widetilde{E}_{L}\right)=0, \\
h^{1}\left(\widetilde{F}_{L}\right) & =h^{1}\left(\widetilde{E}_{L}(-C)\right)=h^{1}\left(\widetilde{E}_{L}\right)=0, \\
h^{0}\left(\widetilde{E}_{L}\right) & =h^{0}(L)+h^{1}(L) .
\end{aligned}
$$

As far as the $C$-slope is concerned, we have

$$
\mu_{C}\left(\widetilde{E}_{L}\right)=2 s .
$$

We will need the following lemma (see also Remark 5.11).

Lemma 2.5. Assume that we are in the Rank-1 case. Let $|L|$ be a $g_{s+2}^{1}$ on $C$. Then $\widetilde{E}_{L}$ is stable. In particular, $M_{v}(S) \neq \emptyset$.

Proof. Observe that the $g_{s+2}^{1}$ is automatically base-point-free because, by Lazarsfeld's proof of Petri's conjecture, the curve $C$ is Petri and thus has no $g_{s+1}^{1}$. Since $\operatorname{Pic}(S)=\mathbb{Z} \cdot[C]$ and $c_{1}\left(\widetilde{E}_{L}\right)=[C]$, we may assume that a destabilizing sequence has the form

$$
0 \longrightarrow \mathcal{O}(n C) \longrightarrow \widetilde{E}_{L} \longrightarrow I_{X}((1-n) C) \longrightarrow 0,
$$

where $n \geqslant 1$ and $X \subset S$ is a zero-dimensional subscheme. But this is absurd since $h^{0}(S, \mathcal{O}(n C))>$ $h^{0}\left(S, \widetilde{E}_{L}\right)=s+2$. The last assertion is obvious.

Definition 2.6. Let $L$ be a globally generated pencil on $C$. The restriction to $C$ of the LazarsfeldMukai bundle $\widetilde{E}_{L}$ is called the Voisin bundle of $L$ and is denoted by the symbol $E_{L}$.

REMARK 2.7. It is a very remarkable fact that the bundle $E_{L}$ depends only on $C, L$ and the first infinitesimal neighborhood $\mathcal{C}_{2}$ of $C$ in $S$. In [Voi92] Voisin proves that, even more generally, one may construct a bundle having all the properties of $E_{L}$ starting from $C, L$ and an embedded ribbon

$$
\mathcal{C}_{2} \subset \mathbb{P}^{g}
$$

having $C$ as hyperplane section. She also observes that the datum of an embedded ribbon $\mathcal{C}_{2} \subset \mathbb{P}^{g}$ having $C$ as hyperplane section is equivalent to the datum of an element $u$ in the kernel of the dual of the Gaussian map

$$
H^{1}\left(C, T_{C}^{2}\right) \longrightarrow H^{1}\left(C, T_{\mathbb{P}^{g-1}} \otimes T_{C}\right)
$$




\section{MUKAI PROGRAM}

Moreover, she proves that if $R_{L} \in H^{0}\left(C, K_{C}+2 L\right)$ is the ramification divisor of the map determined by $|L|$, the class $u R_{L} \in H^{1}\left(C, T_{C}+2 L\right)$ determines an extension

$$
0 \longrightarrow L \longrightarrow E_{L} \longrightarrow K L^{-1} \longrightarrow 0
$$

which splits at the level of cohomology, so that we have

$$
h^{0}\left(E_{L}\right)=h^{0}(L)+h^{1}(L)=h^{1}\left(E_{L}\right) .
$$

From (2.6) it follows that if $L$ a general element of $W_{s+2}^{1}$, then $E_{L}$ is a rank-two vector bundle on $C$ with determinant equal to $K_{C}$ and with $s+2$ linearly independent sections. From the BrillNoether point of view this is most unusual. Certainly a general curve of genus $2 s+1$ admits no such vector bundle. The next section is devoted to the analysis of those Brill-Noether loci that are relevant in our study of curves lying on K3 surfaces.

\section{Brill-Noether loci for moduli of vector bundles on $C$}

Let $(S, C)$ be as in the previous section. We denote by $M_{C}\left(2, K_{C}\right)$ the moduli space of rank-two, semistable vector bundles on $C$ with determinant equal to $K_{C}$. We consider the Brill-Noether locus

$$
M_{C}\left(2, K_{C}, s\right)=\left\{[F] \in M_{C}\left(2, K_{C}\right) \mid h^{0}(C, F) \geqslant s+2\right\}
$$

A point $[F] \in M_{C}\left(2, K_{C}\right)$ corresponding to a stable bundle is smooth for $M_{C}\left(2, K_{C}\right)$ and

$$
T_{[F]}\left(M_{C}\left(2, K_{C}\right)\right)=H^{0}\left(S^{2} F\right)^{\vee} \cong \mathbb{C}^{3 g-3} .
$$

It is well known that the Zariski tangent space to the Brill-Noether locus $M_{C}\left(2, K_{C}, s\right)$ at a point $[F]$ can be expressed in terms of the "Petri" map

$$
\mu: S^{2} H^{0}(F) \longrightarrow H^{0}\left(S^{2} F\right) .
$$

Indeed, we have

$$
T_{[F]}\left(M_{C}\left(2, K_{C}, s\right)\right)=\operatorname{Im}(\mu)^{\perp} .
$$

In particular, $M_{C}\left(2, K_{C}, s\right)$ has expected dimension $3 g-3-\left(\begin{array}{c}s+3 \\ 2\end{array}\right)$.

Also notice that, if $F$ is a rank-two vector bundle on $C$ with determinant equal to $K_{C}$, then since $\chi\left(S^{2} F\right)=\chi\left(F \otimes F^{\vee}\right)-\chi\left(K_{C}\right)=3 g-3$, we get

$$
F \quad \text { stable } \Longrightarrow h^{0}\left(S^{2} F\right)=3 g-3 \quad \Longrightarrow \quad h^{1}\left(S^{2} F\right)=0 \text {. }
$$

Proposition 3.1. Assume that we are in the Rank-1 case. Let $v$ be the Mukai vector (2.1). Then there is a well-defined morphism

$$
\sigma: M_{v}(S) \longrightarrow M_{C}\left(2, K_{C}, s\right), \quad[\mathcal{E}] \longmapsto\left[\mathcal{E}_{\mid C}\right]
$$

Proof. We first show that for every $[\mathcal{E}] \in M_{v}(S)$ the vector bundle $\mathcal{E}_{\mid C}$ is stable. Suppose this is not the case. Then there is an exact sequence

$$
0 \longrightarrow \alpha \longrightarrow \mathcal{E}_{\mid C} \longrightarrow K \alpha^{-1} \longrightarrow 0
$$




\section{E. Arbarello, A. Bruno and E. Sernesi}

with $d=\operatorname{deg} \alpha \geqslant g-1=2 s$. From (3.5), we get a diagram

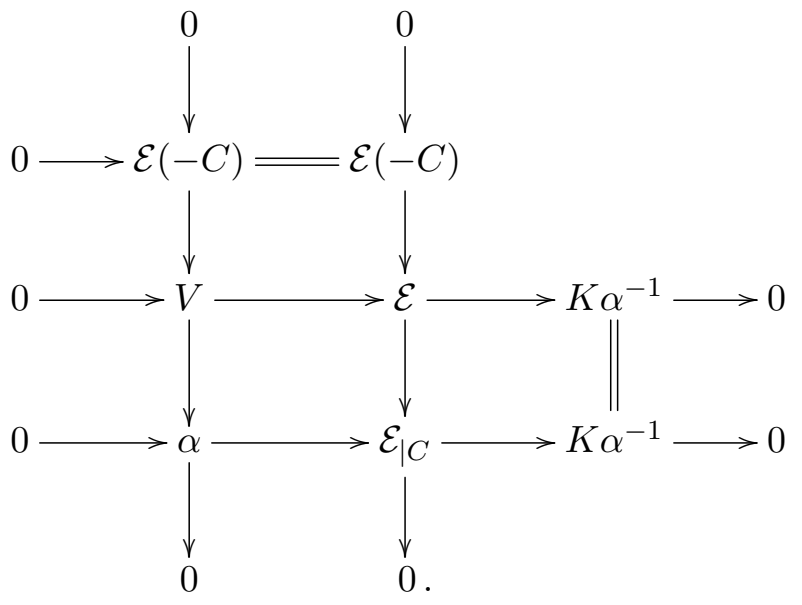

The rank-two locally free sheaf $V$ satisfies

$$
c_{1}(V)=0, \quad c_{2}(V)=c_{2}(\mathcal{E})-c_{1}(\mathcal{E}) \cdot[C]+c_{1}\left(K \alpha^{-1}\right)=s+2-d \leqslant 2-s<0 .
$$

In particular, $V \cong V^{\vee}$, but $V$ is not the trivial bundle. We have

$$
\chi(V)=\chi(\mathcal{E}(-C))+\chi(\alpha)=s+2+d-g+1 \geqslant s+2 .
$$

Thus $2 h^{0}(V) \geqslant s+2$, so that $h^{0}(V) \geqslant 4$. Therefore $V$ cannot be stable, otherwise $\operatorname{dim} \operatorname{End}(V)=$ $h^{0}\left(V \otimes V^{\vee}\right)=h^{0}(V \otimes V)=1$. Suppose that $V$ is strictly semistable, then by the Jordan-Hölder theorem, we have an exact sequence

$$
0 \longrightarrow \mathcal{L} \longrightarrow V \longrightarrow \mathcal{L}^{-1} \otimes I_{X} \longrightarrow 0,
$$

where $X \subset S$ is a zero-dimensional subscheme and $c_{1}(\mathcal{L}) \cdot C=0$. We also have

$$
\chi(V)=\chi(\mathcal{L})+\chi\left(\mathcal{L}^{-1} \otimes I_{X}\right),
$$

so that

$$
0<-c_{2}(V)=c_{1}(\mathcal{L})^{2}-h^{0}\left(\mathcal{O}_{X}\right) \leqslant c_{1}(\mathcal{L})^{2} .
$$

Since $c_{1}(\mathcal{L}) \cdot C=0$ this contradicts the fact that $c_{1}(\mathcal{L})=h C$ for some integer $h$. It follows that $V$ contains a destabilizing sub-line bundle $\mathcal{L}$. Therefore $\mathcal{L}$ satisfies

$$
c_{1}(\mathcal{L}) \cdot C>0, \quad c_{1}(\mathcal{L})^{2} \geqslant-c_{2}(V) \geqslant s-2 .
$$

Then we must have $c_{1}(\mathcal{L})=h C$ for some positive integer $h$, which contradicts the stability of $\mathcal{E}$. We may then conclude that sending $\mathcal{E}$ to $\mathcal{E}_{\mid C}$ gives a well-defined morphism

$$
\sigma^{\prime}: M_{v}(S) \longrightarrow M_{C}\left(2, K_{C}\right) .
$$

By Lemma 2.5, for every pencil $L$ of degree $s+2$, we have

$$
\left[\widetilde{E}_{L}\right] \in M_{v}(S) .
$$

Since, by $(2.3)$, we have $h^{0}\left(S, \widetilde{E}_{L}(-C)\right)=h^{1}\left(S, \widetilde{E}_{L}\right)=h^{2}\left(S, \widetilde{E}_{L}\right)=0$, the same relations must be true for a general point $[\mathcal{E}] \in M_{v}(S)$. Looking then at the exact sequence

$$
0 \longrightarrow \mathcal{E}(-C) \longrightarrow \mathcal{E} \longrightarrow \mathcal{E}_{\mid C} \longrightarrow 0,
$$

we easily see that for a general point $[\mathcal{E}] \in M_{v}(S)$, the point $\left[\mathcal{E}_{\mid C}\right]$ belongs to $M_{C}\left(2, K_{C}, s\right)$, that 


\section{MUKAI PROGRAM}

is, $h^{0}\left(C, \mathcal{E}_{\mid C}\right) \geqslant s+2$. But $M_{v}(S)$ is a smooth K3 surface, in particular it is irreducible. Thus the image of $\sigma^{\prime}$ must be contained in $M_{C}\left(2, K_{C}, s\right)$.

REMARK 3.2. The proof of Proposition 3.1 can be extended to the Rank-2 case using the Hodge Index Theorem but for the last part one needs the existence of a base-point-free pencil $L$ of degree $s+2$ and the stability of $\widetilde{E}_{L}$. The existence of $L$ will be proved in Proposition 4.5 and the stability in Remark 5.11. In Section 5 we will take a different approach to the study of $\sigma: M_{v}(S) \rightarrow M_{C}\left(2, K_{C}, s\right)$ in the Rank-2 case (Corollary 5.8).

The stability of Lazarsfeld-Mukai bundles and Voisin bundles has been studied by various authors. In the Rank-1 case the stability of $\widetilde{E}_{L}$ is almost immediate (Lemma 2.5) but it is also a consequence of a more general result in [Lel13, Section 4]. In the same case the stability of $\widetilde{E}_{L}$, together with Proposition 3.1, gives the stability of its restriction $E_{L}$. This particular result is also a consequence of [AFO13, Theorem 4.1]. The stability of both $\widetilde{E}_{L}$ and $E_{L}$ cannot be deduced from the papers mentioned above.

Finally, we want to study the differential of the morphism $\sigma: M_{v}(S) \rightarrow M_{C}\left(2, K_{C}, s\right)$ whenever it is well defined.

Proposition 3.3. Assume that the morphism

$$
\sigma: M_{v}(S) \longrightarrow M_{C}\left(2, K_{C}, s\right), \quad \mathcal{E} \longmapsto \mathcal{E}_{\mid C}
$$

is well defined. Look at the composition

$$
\sigma^{\prime}: M_{v}(S) \stackrel{\sigma}{\longrightarrow} M_{C}\left(2, K_{C}, s\right) \stackrel{j}{\longrightarrow} M_{C}\left(2, K_{C}\right),
$$

where $j$ is the inclusion. Let $\mathcal{E} \in M_{v}(S)$ and consider the following conditions:

(i) $H^{1}\left(S, S^{2} \mathcal{E}\right)=0$.

(ii) $H^{0}\left(S, S^{2} \mathcal{E}(-C)\right)=0$.

(iii) The map $S^{2} H^{0}(S, \mathcal{E}) \rightarrow H^{0}\left(S, S^{2} \mathcal{E}\right)$ is surjective.

Then

(1) if condition (i) is satisfied, $d \sigma^{\prime}$ is injective at $[\mathcal{E}]$;

(2) if conditions (i), (ii) and (iii) are satisfied, $M_{C}\left(2, K_{C}, s\right)$ is non-singular of dimension two at $\mathcal{E}_{\mid C}$ and $\sigma$ is étale at $\mathcal{E}$.

Proof. (1) Write $E=\mathcal{E}_{\mid C}$. We use the isomorphism

$$
\mathcal{E}^{\vee} \otimes \mathcal{E}=\mathcal{E}^{\vee} \otimes \mathcal{E}^{\vee}(C) \cong\left(S^{2} \mathcal{E}^{\vee} \oplus \wedge^{2} \mathcal{E}^{\vee}\right)(C) \cong S^{2} \mathcal{E}^{\vee}(C) \oplus \mathcal{O}_{S}
$$

We have

$$
\begin{aligned}
T_{[\mathcal{E}]}\left(M_{v}(S)\right) & =H^{1}\left(S, \mathcal{E} \otimes \mathcal{E}^{\vee}\right)=H^{1}\left(S, S^{2} \mathcal{E}^{\vee}(C)\right), \\
T_{[E]}\left(M_{C}\left(2, K_{C}\right)\right) & =H^{0}\left(C, S^{2} E\right)^{\vee}=H^{1}\left(C, S^{2} E^{\vee}\left(K_{C}\right)\right) .
\end{aligned}
$$

Thus $d \sigma^{\prime}$ is the restriction homomorphism

$$
d \sigma^{\prime}: H^{1}\left(S, S^{2} \mathcal{E}^{\vee}(C)\right) \longrightarrow H^{1}\left(C, S^{2} E^{\vee}\left(K_{C}\right)\right) .
$$

It follows that

$$
\operatorname{ker} \sigma^{\prime}=H^{1}\left(S, S^{2} \mathcal{E}^{\vee}\right)=H^{1}\left(S, S^{2} \mathcal{E}\right) \text {. }
$$




\section{E. Arbarello, A. Bruno and E. Sernesi}

(2) Consider the following commutative diagram of maps:

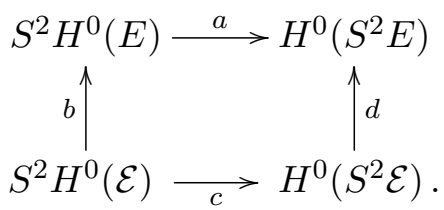

Condition (ii) implies that $d$ is injective and condition (iii) implies that the map $c$ is surjective. Therefore

$$
\operatorname{corank}(a) \leqslant \operatorname{corank}(d) \leqslant h^{1}\left(S^{2} \mathcal{E}(-C)\right) .
$$

Now consider the decomposition (3.8). Since $\mathcal{E}$ is stable we have

$$
h^{0}\left(\mathcal{E}^{\vee} \otimes \mathcal{E}\right)=1 \quad \text { and } \quad h^{1}\left(\mathcal{E}^{\vee} \otimes \mathcal{E}\right)=2
$$

and from the decomposition we deduce that $h^{1}\left(S^{2} \mathcal{E}(-C)\right)=2$ as well, using hypothesis (ii). Therefore $\operatorname{corank}(a) \leqslant 2$; since $\operatorname{coker}(a)^{\perp}=T_{[E]} M_{C}\left(2, K_{C}, s\right)$, we conclude that

$$
\operatorname{dim}\left[T_{[E]} M_{C}\left(2, K_{C}, s\right)\right] \leqslant 2 .
$$

But from condition (i) and part (1), it follows that $M_{C}\left(2, K_{C}, s\right)$ has dimension at least two at $[E]$. This proves part (2).

\section{Geometry of $(S, C)$ in the Rank-2 case}

As in the previous two sections we denote by $(S, C)$ a pair consisting of a K3 surface $S$ and a smooth curve $C \subset S$ of genus

$$
g(C)=2 s+1, \quad s \geqslant 5 .
$$

In this section we assume that we are in the Rank- 2 case and we prove a few technical results.

Lemma 4.1. (i) We have $h^{0}(S, \mathcal{O}(n A+m B))=0$ whenever $n<0$.

(ii) We have $h^{i}(S, \mathcal{O}(A-B))=h^{i}(S, \mathcal{O}(B-A))=0$ for all $i$.

(iii) Every element in $|A|$ is integral and has Clifford index at least two. In particular, $|A|$ is very ample.

Proof. (i) This is immediate by restricting to $B$.

(ii) We have $(A-B)^{2}=-4$ and, by part (i), we also have $h^{2}(S, \mathcal{O}(A-B))=0$. Hence $h^{1}\left(S, \mathcal{O}(A-B)=h^{0}(S, \mathcal{O}(A-B))\right.$. Suppose $h^{0}(S, \mathcal{O}(A-B)) \neq 0$. Let $D$ be an effective divisor linearly equivalent to $A-B$. If $D$ is connected, then by [Sai74, Lemma 2.2], we get $h^{1}(S, \mathcal{O}(D))=0$ and we are done. Otherwise, we have

$$
\begin{gathered}
D=D_{1}+D_{2}, \quad D_{1} \cdot D_{2}=0, \quad h^{0}\left(S, \mathcal{O}\left(D_{i}\right)\right) \geqslant 1, \quad i=1,2 . \\
D_{1}=n A+m B, \quad D_{2}=h A+k B, \quad n+h=1, \quad m+k=-1 .
\end{gathered}
$$

Since $D_{1}$ and $D_{2}$ are effective, by statement (i), we must have $n \geqslant 0, h \geqslant 0$. Thus either $n=1$ and $h=0$, or $n=0$ and $h=1$. In any event, we would get $D_{1} \cdot D_{2} \neq 0$, which is absurd.

(iii) Let us prove the first part of statement (iii). Write $A=\Gamma+\Delta$, with both $\Gamma$ and $\Delta$ effective. Then $\Gamma=n A+m B$. Since $A \cdot B \geqslant \Gamma \cdot B=n A \cdot B$, we get $n \leqslant 1$. Since $h^{0}(S, \mathcal{O}(\Gamma)) \neq 0$, by part (i) we must have $n=0,1$. If $n=0$, then $m B$ must be a subcurve of $A$, which goes against part (ii). If $n=1$, then since $\Gamma$ is a subcurve of $A$, we must have $m<0$, which again 


\section{MUKAI PROGRAM}

violates part (ii).

Let us now prove the second part of statement (iii). By the main theorem of [GL87] and by its refinement in [BFT10], all curves $A^{\prime} \in|A|$ have the same Clifford index and Cliff $\left(A^{\prime}\right)$ is computed by the restriction to $A^{\prime}$ of an invertible sheaf $L$ on $S$. So, in order to complete the proof of part (iii) we must exclude the existence of $L \in \operatorname{Pic}(S)$ with any of the following properties:

(1) We have $L^{2}=0$ and $L \cdot A^{\prime}=2$ (that is, $A^{\prime}$ is hyperelliptic).

(2) We have $L^{2}=0$ and $L \cdot A^{\prime}=3$ (that is, $A^{\prime}$ is trigonal).

(3) We have $s=6, L^{2}=2$ and $L \cdot A=5$ (that is, $A^{\prime}$ is isomorphic to a non-singular plane quintic).

Let us consider property (2). We must have $L=n A+m B$ and the two conditions translate into

$$
(n A+m B)^{2}=2 n^{2}(s-1)+2 n m(s+1)=0 \quad \text { and } \quad 2 n(s-1)+m(s+1)=3 \quad(s \geqslant 5),
$$

which are clearly incompatible. The hyperelliptic case (1) is similar.

In case (3), we must have

$$
(n A+m B)^{2}=2 n(5 n+7 m)=2 \text { and } 10 n+7 m=5,
$$

implying the impossible identity $5 n(n+1)=2$.

REMARK 4.2. (a) From point (iii) of Lemma 4.1, it follows that, via the linear system $|A|$, the surface $S$ is embedded in $\mathbb{P}^{S}$ as a projectively normal surface whose ideal is generated by quadrics.

(b) Let $I_{S}$ and $I_{C}$ be the ideal sheaves of $S$ and $C$, respectively, in $\mathbb{P}^{s}$. Recall that $2 A-C \sim$ $A-B$ as divisors on $S$. From point (a) and statement (ii) of Lemma 4.1, we deduce that $H^{0}(S, \mathcal{O}(2 A)) \cong H^{0}(C, 2 \eta)$ and, in particular, we get a surjection

$$
m: S^{2} H^{0}(C, \eta) \longrightarrow H^{0}(C, 2 \eta) \longrightarrow 0
$$

and an equality

$$
H^{0}\left(S, I_{S}(2)\right)=H^{0}\left(C, I_{C}(2)\right)=\operatorname{ker}(m) .
$$

Lemma 4.3. Let $D \subset S$ be a finite closed subscheme of length $d \geqslant 1$. Assume

$$
h^{0}\left(S, \mathcal{I}_{D}(A)\right) \geqslant \max \left\{3, s-\frac{d-1}{2}\right\}
$$

Then $d=1$.

Proof. We view $S$ as embedded in $\mathbb{P}^{s}=\mathbb{P} H^{0}(S, \mathcal{O}(A))^{\vee}$. Consider a hyperplane $H$ passing through $D$, that is, defining a non-zero element of $H^{0}\left(S, \mathcal{I}_{D}(A)\right)$. We set $A=H \cap S$. We may view $D$ as a subscheme of the integral curve $A$. As such it defines a rank-one torsion free sheaf on $A$ which we still denote by $D$. From (4.3) we get

$$
h^{0}\left(A, \omega_{A}(-D)\right) \geqslant 2 .
$$

Thus, by the Riemann-Roch theorem on $A$, we have

$$
h^{0}\left(A, \mathcal{O}_{A}(D)\right)=h^{0}\left(A, \omega_{A}(-D)\right)+d-s+1 \geqslant \frac{d+1}{2} .
$$

Therefore either $h^{0}\left(A, \mathcal{O}_{A}(D)\right)=1$ and $d+1 \leqslant 2$, implying that $d \leqslant 1$, which is precisely what we aim at, or $h^{0}\left(A, \mathcal{O}_{A}(D)\right) \geqslant 2$, which, together with (4.4), tells us that $D$ contributes to the 


\section{E. Arbarello, A. Bruno and E. Sernesi}

Clifford index of $A$. Let us show that this case cannot occur. By (4.5) we get

$$
\text { Cliff } \begin{aligned}
D & =d-2 h^{0}\left(A, \mathcal{O}_{A}(D)\right)+2 \\
& \leqslant d-2\left(\frac{d+1}{2}\right)+2 \leqslant 1 .
\end{aligned}
$$

This implies that $\operatorname{Cliff}(A) \leqslant 1$, contradicting Lemma 4.1 (iii).

REMARK 4.4. In a sense, the technical lemma we just proved is our substitute for Mukai's Lemma 7, which is ubiquitous in [Muk96].

It suggests the possibility of introducing the notion of Clifford index of a zero-dimensional closed subscheme $D$ on a polarized $\mathrm{K} 3$ surface $(S, H)$ by letting

$$
\operatorname{Cliff}(D)=2 g-d-2 h^{0}\left(S, \mathcal{I}_{D}(H)\right)+2,
$$

where $d=\operatorname{length}(D)$ and $g=\frac{1}{2} H^{2}+1$ is the genus of $H$. One says that $D$ contributes to the Clifford index of $H$ if both $h^{0}\left(S, \mathcal{I}_{D}(H)\right) \geqslant 3$ and $h^{0}\left(S, \mathcal{I}_{D}(H)\right)+d-g+1 \geqslant 3$. A straightforward generalization of the proof of Lemma 4.3 gives that if all $H^{\prime} \in|H|$ are integral, then

$$
\operatorname{Cliff}(H)=\min \{\operatorname{Cliff}(D): D \subset S \text { contributes to } \operatorname{Cliff}(H)\} .
$$

Our next aim is to prove the following proposition regarding linear series of degree $s+2$ on $C$ in the Rank-2 case.

Proposition 4.5. (i) Let $|L|$ be a degree- $(s+2)$, base-point-free pencil on $C \subset S$. Then $L$ is Petri.

(ii) We have $h^{0}\left(C, \eta \xi^{-1}\right)=1$, and thus $\xi$ is a smooth isolated point of $W_{s+1}^{1}$.

(iii) There exists a base-point-free $g_{s+2}^{1}$ on $C$.

We are going to use the following lemma due to Green-Lazarsfeld and Donagi-Morrison. We take its statement from [CP95, Lemma 2.1].

Lemma 4.6 (Green-Lazarsfeld and Donagi-Morrison). Let $S$ be a K3 surface. Let $|L|$ be a basepoint-free pencil on a smooth curve $C$ lying on $S$. If the Lazarsfeld-Mukai bundle $\widetilde{E}_{L}$ is not simple (that is, it has non-trivial automorphisms), then there exist line bundles $M$ and $N$ on $S$ and a zero-dimensional subscheme $X \subset S$ such that

(i) $h^{0}(S, M) \geqslant 2, h^{0}(S, N) \geqslant 2$;

(ii) the line bundle $N$ is base-point-free;

(iii) there is an exact sequence

$$
0 \longrightarrow M \longrightarrow \widetilde{E}_{L} \longrightarrow N \otimes I_{X} \longrightarrow 0 .
$$

Moreover, if $h^{0}(S, M-N)=0$, then $\operatorname{Supp}(X)=\emptyset$ and sequence (4.7) splits.

Proof of Proposition 4.5. As far as point (i) is concerned, we proceed exactly as in Lazarsfeld's proof of Petri's conjecture. It is then enough to prove that $\widetilde{E}_{L}$ is simple. Suppose it is not. By Lemma 4.6, there is an exact sequence (4.7). Since $\operatorname{Pic}(S)=\mathbb{Z} \cdot[A] \oplus \mathbb{Z} \cdot[B], C=A+B$ and $c_{1}\left(\widetilde{E}_{L}\right)=C$, we must have

$$
M=m A+n B, \quad N=h A+k B, \quad(m+h-1) A+(n+k-1) B \sim 0
$$




\section{MUKAI PROGRAM}

from condition (i) of the lemma, and from Lemma 4.1, we get that the pair $\{M, N\}$ coincides with the pair $\{A, B\}$. On the other hand, by Lemma 4.1, we have $H^{0}(A-B)=0$. Using Lemma 4.6 again, sequence (4.7) splits and $\widetilde{E}_{L}=\mathcal{O}(A) \oplus \mathcal{O}(B)$. This is absurd since $h^{0}\left(S, \widetilde{E}_{L}\right)=s+2$.

Regarding item (ii), looking at the Petri map $H^{0}(C, \xi) \otimes H^{0}(C, \eta) \rightarrow H^{0}\left(C, \omega_{C}\right)$, and using the base-point-free-pencil trick, we see that $h^{0}\left(C, \eta \xi^{-1}\right) \geqslant 1$. On the other hand, looking at the exact sequence

$$
0 \longrightarrow \mathcal{O}_{S}(-2 B) \longrightarrow \mathcal{O}_{S}(A-B) \longrightarrow \eta \xi^{-1} \longrightarrow 0,
$$

we see that $h^{0}\left(C, \eta \xi^{-1}\right) \leqslant 1$.

As far as point (iii) is concerned, consider the smooth locus

$$
V=\{\xi(p) \mid p \in C\} \subset W_{s+2}^{1} .
$$

To analyze $W_{s+2}^{1}$ along $V$, we look at the Petri map

$$
\mu_{0, \xi(p)}: H^{0}(C, \xi(p)) \otimes H^{0}(C, \eta(-p)) \longrightarrow H^{0}\left(C, K_{C}\right) .
$$

By assumption, we have $H^{0}(C, \xi(p))=H^{0}(C, \xi)$ and

$$
\operatorname{ker} \mu_{0, \xi(p)}=H^{0}\left(C, \eta \xi^{-1}(-p)\right) \text {. }
$$

By part (ii), we know that $h^{0}\left(C, \eta \xi^{-1}\right)=1$. Let $D \neq 0$ be the divisor of a non-zero section of $\eta \xi^{-1}$. By Brill- Noether theory, it follows that $V$ is a one-dimensional component of $W_{s+2}^{1}$ and that $\xi(p)$ is a singular point of $W_{s+2}^{1}$ if and only if $p \in \operatorname{Supp} D$. Moreover, in this case

$$
\operatorname{dim} T_{\xi(p)} W_{s+2}^{1}=2 .
$$

A priori it could be that for $p \in \operatorname{Supp} D$, the dimension of $W_{s+2}^{1}$ at $\xi(p)$ is equal to one and $\xi(p)$ is an embedded point. However, for a determinantal variety of the correct dimension this cannot be the case. Thus there must be a one-dimensional component $V^{\prime}$ of $W_{s+2}^{1}$, distinct from $V$ and meeting $V$ at $\xi(p)$. Now suppose that there is no base-point-free $g_{s+2}^{1}$ in $V^{\prime}$. Then

$$
V^{\prime}=\left\{\xi^{\prime}\left(p^{\prime}\right) \mid p^{\prime} \in C\right\}
$$

for some fixed $\xi^{\prime} \in W_{s+1}^{1}$ with $\xi^{\prime} \neq \xi$. Therefore there is a point $p^{\prime} \in C$ such that

$$
\xi(p)=\xi^{\prime}\left(p^{\prime}\right) .
$$

But then $\xi=\xi^{\prime}$, contrary to the assumption.

Proposition 4.5, together with the results in [AC81], gives the following result.

Proposition 4.7. Set $g=2 s+1$. Let $M_{g, s+1}^{1} \subset M_{g}$ be the irreducible divisor of curves possessing a $g_{s+1}^{1}$. Let $[C]$ be a general point in $M_{g, s+1}^{1}$. Then there is a unique $g_{s+1}^{1}$ on $C$ and every degree$(s+2)$ base-point-free pencil on $C$ satisfies Petri's condition.

\section{Brill-Noether loci in the Rank-2 case}

In this section we assume that we are in the Rank-2 case. Using Lemma 4.1(ii) and proceeding as in [Muk96, Section 3], with each point $x \in S$ we associate a rank-two, pure sheaf $\mathcal{E}_{x}$ defined as the unique extension

$$
0 \longrightarrow \mathcal{O}_{S}(B) \longrightarrow \mathcal{E}_{x} \longrightarrow I_{x}(A) \longrightarrow 0
$$

We set $E_{x}=\mathcal{E}_{x \mid C}$. 


\section{E. Arbarello, A. Bruno and E. Sernesi}

The main theorem we want to prove in this section is the following generalization of [Muk96, Theorem 3].

Theorem 5.1. By associating with $x \in S$ the bundle $E_{x}$ on $C$, we obtain an isomorphism between $S$ and $M_{C}\left(2, K_{C}, s\right)$.

The proof of the theorem will be obtained from the following chain of facts to be proved.

- Both $E_{x}$ and $\mathcal{E}_{x}$ are stable. Moreover, $\left[\mathcal{E}_{x}\right] \in M_{v}(S)$ and $\left[E_{x}\right] \in M_{C}\left(2, K_{C}, s\right)$.

- The map

$$
\rho: S \longrightarrow M_{v}(S), \quad x \longmapsto\left[\mathcal{E}_{x}\right]
$$

is an isomorphism of $\mathrm{K} 3$ surfaces.

- From these two facts it follows that the morphism

$$
\sigma: M_{v}(S) \longrightarrow M_{C}\left(2, K_{C}, s\right), \quad[\mathcal{E}] \longmapsto\left[\mathcal{E}_{\mid C}\right]
$$

is well defined.

- The map $\sigma$ is bijective.

- For every $x \in S$,

$$
\operatorname{dim} T_{\left[E_{x}\right]}\left(M_{C}\left(2, K_{C}, s\right)\right)=2
$$

and the differential of $\sigma$ is an isomorphism at $\left[\mathcal{E}_{x}\right]$.

The last two items finally give the following result.

- The map $\sigma$ is, in fact, an isomorphism of smooth K3 surfaces and therefore the map $\sigma \rho: S \rightarrow M_{C}\left(2, K_{C}, s\right)$ is an isomorphism.

We need to establish a number of preliminary results. First of all, since $H^{1}(S, \mathcal{O}(B))=0$, (5.1) gives the exact sequence

$$
\begin{gathered}
0 \longrightarrow H^{0}\left(S, \mathcal{O}_{S}(B)\right) \longrightarrow H^{0}\left(S, \mathcal{E}_{x}\right) \longrightarrow H^{0}\left(S, I_{x}(A)\right) \longrightarrow 0, \\
H^{0}\left(S, \mathcal{E}_{x}\right)=s+2, \quad H^{i}\left(S, \mathcal{E}_{x}\right)=0, \quad i=1,2
\end{gathered}
$$

and $\mathcal{E}_{x}$ is generated by global sections. Notice that since $\operatorname{det} \mathcal{E}_{x}=\mathcal{O}(C)$, we have

$$
\mathcal{E}_{x}^{\vee} \cong \mathcal{E}_{x}(-C) \text {. }
$$

From (5.6), we get

$$
0 \longrightarrow \mathcal{E}_{x}^{\vee} \longrightarrow \mathcal{E}_{x} \longrightarrow E_{x} \longrightarrow 0
$$

We then get an isomorphism

$$
H^{0}\left(S, \mathcal{E}_{x}\right) \cong H^{0}\left(C, E_{x}\right)
$$

and, in particular,

$$
\operatorname{dim} H^{0}\left(C, E_{x}\right)=s+2=h^{0}(C, \xi)+h^{0}(C, \eta)-1 .
$$

If $x \notin C$, restricting (5.1) to $C$ gives

$$
0 \longrightarrow \xi \longrightarrow E_{x} \longrightarrow \eta \longrightarrow 0 .
$$

If $x \in C$, then factoring out the torsion from $I_{x}(A) \otimes \mathcal{O}_{C}$, we get

$$
0 \longrightarrow \xi(x) \longrightarrow E_{x} \longrightarrow \eta(-x) \longrightarrow 0 \text {. }
$$




\section{MUKAI PROGRAM}

We are now going to prove two results that are key elements in the proof of the stability of $E_{x}$ (Proposition 5.5).

Lemma 5.2. The extension (5.11) is non-split.

Proof. To prove this we proceed as in the proof of [Muk96, Proposition 3].

Let $E_{x} \rightarrow \xi(x)$ be a splitting. Consider the diagram

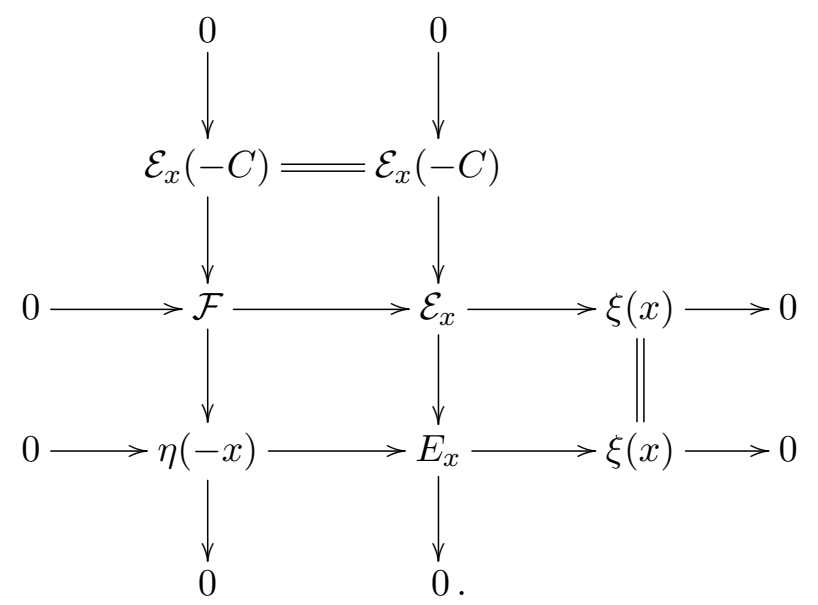

Then $H^{0}(S, \mathcal{F})$ is an $s$-dimensional subspace of $H^{0}\left(S, \mathcal{E}_{x}\right)$ mapping onto $H^{0}\left(S, I_{x} A\right)$. We have $c_{1}(\mathcal{F})=0$ so that $\wedge^{2} \mathcal{F}=\mathcal{O}_{S}$. Look at the evaluation map $H^{0}(S, \mathcal{F}) \otimes \mathcal{O}_{S} \rightarrow \mathcal{F} \subset \mathcal{E}_{x}$ and take its second wedge product

$$
\wedge^{2}\left(H^{0}(S, \mathcal{F}) \otimes \mathcal{O}_{S}\right) \stackrel{\alpha}{\longrightarrow} \wedge^{2} \mathcal{F}=\mathcal{O}_{S} \subset \wedge^{2} \mathcal{E}_{x}=\mathcal{O}_{S}(C)
$$

where $\alpha=\left(c_{1}, \ldots, c_{n}\right)$ with $c_{i} \in \mathbb{C}, i=1, \ldots, n$. Restricting $\alpha$ to $C$ we see that it vanishes on $x$ and therefore vanishes identically. Thus the image of the evaluation map $H^{0}(S, \mathcal{F}) \otimes \mathcal{O}_{S} \rightarrow \mathcal{F} \subset \mathcal{E}_{x}$ is of rank one and is isomorphic to $I_{x}(A)$, which is a contradiction, since $\mathcal{E}_{x}$ is non-split.

Lemma 5.3. Let $E$ be a rank-two vector bundle (not necessarily semi-stable) with canonical determinant on $C$. Suppose that $h^{0}(E)=s+2$ and that $E$ contains a sub-line bundle isomorphic to $\xi$ or $\xi(p)$ for a point $p \in C$. Then one of the following holds:

(i) The bundle $E$ is stable.

(ii) There is an exact sequence $0 \rightarrow \xi \rightarrow E \rightarrow \eta \rightarrow 0$, and $\eta(-p)$ is a destabilizing subsheaf of $E$.

(iii) $E=\xi(p) \oplus \eta(-p)$.

Proof. Assume that $E$ is not stable; then we must have an exact sequence

$$
0 \longrightarrow \alpha \longrightarrow E \longrightarrow \beta \longrightarrow 0,
$$

where $\alpha$ is a line bundle on $C$ of degree greater than or equal to $g-1$. We have two possible 
diagrams:
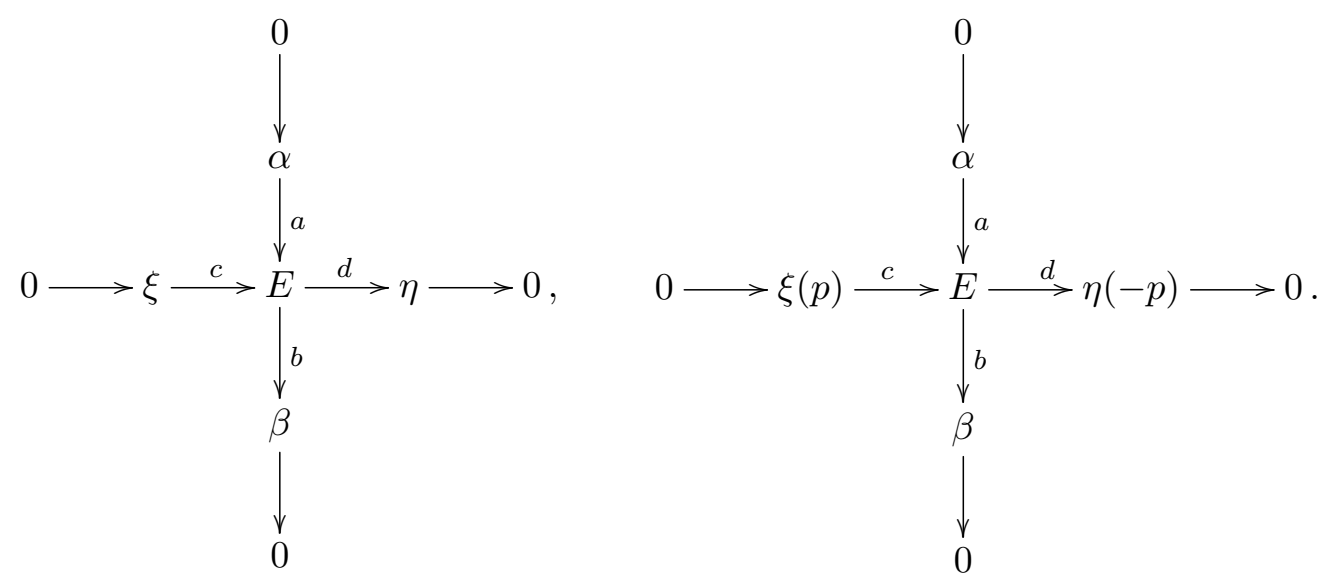

In the first case, since $\operatorname{deg} \alpha \geqslant 2 s>s+1=\operatorname{deg} \xi$, there can be no injective map from $\alpha$ to $\xi$. In the second case, since $\operatorname{deg} \alpha \geqslant 2 s>s+2=\operatorname{deg} \xi(p)$, there can be no injective map from $\alpha$ to $\xi(p)$. Thus, we must have $\alpha=\eta(-D)$ and $\beta=\xi(D)$ for some positive divisor of degree $d \leqslant s-1$ or $d \leqslant s-2$, respectively. We have

$$
\begin{aligned}
s+2=h^{0}(E) & \leqslant h^{0}(\xi(D))+h^{0}(\eta(-D)) \\
& =2 h^{0}(\eta(-D))+s+1+d-g+1 \\
& =2 h^{0}(\eta(-D))-s+1+d .
\end{aligned}
$$

It follows that

Consequently, we have

$$
h^{0}(\eta(-D)) \geqslant s-\frac{d-1}{2}
$$

$$
h^{0}(\eta(-D)) \geqslant s-\frac{d-1}{2} \geqslant \frac{s+2}{2} \geqslant 3 \quad \text { or } \quad h^{0}(\eta(-D)) \geqslant \frac{s+3}{2} \geqslant 3,
$$

respectively, since $s \geqslant 5$. We can then apply Lemma 4.3 and we get $d \leqslant 1$. Then the only possibilities are the ones described in statements (ii) and (iii).

The next result needed to prove the stability of $\mathcal{E}_{x}$ and $E_{x}$ is [Muk96, Lemma 2]. We include its statement for the convenience of the reader.

Lemma 5.4 (Mukai). Let $L$ be a line bundle on a smooth curve $C$ and consider non-trivial extensions

$$
0 \longrightarrow L \longrightarrow E \longrightarrow M \longrightarrow 0
$$

with $M=K_{C} L^{-1}$.

(i) The extensions $E$ with $h^{0}(E)=h^{0}(L)+h^{0}(M)$ are parametrized by the projective space $\mathbb{P}^{*}$ Coker $\left\{S^{2} H^{0}(M) \rightarrow H^{0}\left(M^{2}\right)\right\}$.

(ii) Assume that the multiplication map $S^{2} H^{0}(M) \rightarrow H^{0}\left(M^{2}\right)$ is surjective. Then $h^{0}(C, E) \leqslant$ $h^{0}(C, L)+h^{0}(C, M)-1$. Moreover, the non-trivial extensions $E$ such that $h^{0}(C, E)=h^{0}(C, L)+$ $h^{0}(C, M)-1$ are parametrized by the quadratic hull of the image of $\Phi_{|M|}: C \rightarrow \mathbb{P}^{*} H^{0}(C, M)$. More precisely, for every point $x$ of the quadric hull, there is a unique extension $E$ such that the image of the linear map $H^{0}(C, E) \rightarrow H^{0}(C, M)$ is the codimension one subspace corresponding to $x$. 


\section{MUKAI PROGRAM}

We are now ready to prove the following result.

Proposition 5.5. Both $E_{x}$ and $\mathcal{E}_{x}$ are stable. Moreover, $\left[\mathcal{E}_{x}\right] \in M_{v}(S)$ and $\left[E_{x}\right] \in M(C, K, s)$.

Proof. Assume first that $x \notin C$. We are then in case (5.10). By (5.9) the sequence cannot split. By Lemma 5.3, if $E_{x}$ is not stable, then it sits in an exact sequence

$$
0 \longrightarrow \eta(-p) \longrightarrow E_{x} \longrightarrow \xi(p) \longrightarrow 0
$$

for some $p \in C$ and we have $H^{0}\left(C, E_{x}\right) \cong H^{0}(C, \xi(p)) \oplus H^{0}(C, \eta(-p))=H^{0}(C, \xi) \oplus H^{0}(C, \eta(-p))$. Thus the codimension one subspace of $H^{0}(C, \eta)$ is $H^{0}(C, \eta(-p))$. But then $x=p \in C$, which gives a contradiction.

Let then $x \in C$. Then we are in case (5.11). By Lemma 5.2, this sequence cannot split. The stability of $E_{x}$ now follows immediately from Lemma 5.3.

The stability of $\mathcal{E}_{x}$ is now clear. If $L$ is a destabilizing subsheaf of $\mathcal{E}_{x}$, then $L \cdot C \geqslant C^{2} / 2$. But then $L_{\mid C}$ would destabilize $E_{x}$. The last assertion is a consequence of (5.5) and (5.9).

REMARK 5.6. From the preceding arguments we learned that points $x$ in the quadratic hull of $C \subset \mathbb{P}^{s}$, not belonging to $C$, correspond to extensions of type (5.10) where $E_{x}$ is a stable bundle. On the other hand, points $x$ belonging to $C$ correspond to extensions of type (a):

$$
0 \longrightarrow \xi \longrightarrow E_{x}^{\prime} \longrightarrow \eta \longrightarrow 0,
$$

where $E_{x}^{\prime}$ is destabilized by $\eta(-x)$. Finally, if $D$ is the divisor of a section of $\eta \xi^{-1}$, a point $x \in \operatorname{Supp}(D)$ corresponds to an extension of type (b):

$$
0 \longrightarrow \xi \longrightarrow E_{x}^{\prime \prime}=\eta(-x) \oplus \xi(x) \longrightarrow \eta \longrightarrow 0
$$

and $E_{x}^{\prime \prime}$ is clearly unstable. In both cases (a) and (b), the "stable limit" replacing $E_{x}^{\prime}$ and $E_{x}^{\prime \prime}$, respectively, is $E_{x}$ as in (5.11).

We next come to the following result.

Proposition 5.7. The map

$$
\rho: S \longrightarrow M_{v}(S), \quad x \longmapsto\left[E_{x}\right]
$$

is an isomorphism of K3 surfaces.

Proof. We again follow Mukai's line of reasoning [Muk96, p. 194 before Lemma 8, and p. 195 after Lemma 11]. The local to global spectral sequence of ext gives

$$
H^{j}\left(S, \mathcal{E} x t_{\mathcal{O}_{S}}^{i}\left(I_{x}(A), \mathcal{O}_{S}(B)\right)\right) \Rightarrow \operatorname{Ext}_{\mathcal{O}_{S}}^{i+j}\left(I_{x}(A), \mathcal{O}_{S}(B)\right) .
$$

By Lemma 4.1, the natural map

$$
\operatorname{Ext}_{\mathcal{O}_{S}}^{1}\left(I_{x}(A), \mathcal{O}_{S}(B)\right) \longrightarrow H^{0}\left(S, \mathcal{E} x t_{\mathcal{O}_{S}}^{1}\left(I_{x}(A), \mathcal{O}_{S}(B)\right)\right) \cong \mathbb{C}
$$

is an isomorphism so that the extension (5.1) is the unique non-trivial extension of $I_{x}(A)$ by $\mathcal{O}_{S}(B)$. Now one can perform a relative version of this construction. We let $T$ be a copy of $S$ and let $\Delta$ be the diagonal of $S \times T$. We have an isomorphism

$$
\left.\mathcal{E} x t_{\mathcal{O}_{S \times T}}^{1}\left(I_{\Delta}\left(p^{*} A\right), \mathcal{O}_{S \times T}\left(q^{*} B\right)\right) \longrightarrow q_{*} \mathcal{E} t_{\mathcal{O}_{S \times T}}^{1}\left(I_{\Delta}\left(p^{*} A\right), \mathcal{O}_{S \times T}\left(q^{*} B\right)\right)\right) \cong \mathcal{O}_{T}(B-A)
$$

which is a relative version of (5.16). We then have a universal extension

$$
0 \longrightarrow \mathcal{O}_{S \times S}\left(p^{*} B\right) \longrightarrow \mathcal{F} \longrightarrow I_{\Delta}\left(p^{*} A+q^{*}(B-A)\right) \longrightarrow 0
$$




\section{E. Arbarello, A. Bruno and E. Sernesi}

whose restriction to $S \times\{x\}$ is (5.1). This gives a well-defined morphism

$$
\rho: S \longrightarrow M_{v}(S), \quad x \longmapsto\left[\mathcal{E}_{x}\right] .
$$

As $S$ and $M_{v}(S)$ are smooth K3 surfaces, to prove that $\rho$ is an isomorphism it suffices to show that it is injective and for this it suffices to show that

$$
\operatorname{dim} \operatorname{Hom}\left(O(B), \mathcal{E}_{x}\right)=1, \quad \text { that is, } \quad h^{0}\left(S, \mathcal{E}_{x}(-B)\right)=1 .
$$

But this follows readily from the exact sequence

$$
0 \longrightarrow \mathcal{O}_{S} \longrightarrow \mathcal{E}_{x}(-B) \longrightarrow I_{x}(A-B) \longrightarrow 0 .
$$

Corollary 5.8. The map $\sigma: M_{v}(S) \longrightarrow M_{C}\left(2, K_{C}, s\right)$ is well defined.

Proof. The corollary is an immediate consequence of Propositions 5.5 and 5.7.

Proposition 5.9. The map $\sigma$ is bijective.

Proof. The map $\sigma$ is injective. Clearly what we have to prove is that $\operatorname{dim} \operatorname{Hom}\left(\xi, E_{x}\right)=1$ or, in other words, that $h^{0}\left(C, E_{x} \xi^{-1}\right)=1$. From the exact sequence

$$
0 \longrightarrow \mathcal{O}_{S}(-B-C) \longrightarrow \mathcal{E}_{x}(-B) \longrightarrow E_{x} \xi^{-1} \longrightarrow 0
$$

we get

$$
H^{0}\left(S, \mathcal{E}_{x}(-B)\right) \cong H^{0}\left(C, E_{x} \xi^{-1}\right)
$$

From the sequence

$$
0 \longrightarrow \mathcal{O}_{S} \longrightarrow \mathcal{E}_{x}(-B) \longrightarrow I_{x}(A-B) \longrightarrow 0,
$$

we get $H^{0}\left(S, \mathcal{E}_{x}(-B)\right) \cong \mathbb{C}$.

The map $\sigma$ is surjective. Let $[E] \in M_{C}\left(2, K_{C}, s\right)$. Let us recall Lemma 1 of [Muk96]. Again, we include its statement for the convenience of the reader.

Lemma 5.10 (Mukai). Let $E$ be a rank-two vector bundle of canonical determinant $\zeta$ a line bundle on $C$. If $\zeta$ is generated by global sections, then we have

$$
\operatorname{dim} \operatorname{Hom}_{\mathcal{O}_{C}}(\zeta, E) \geqslant h^{0}(E)-\operatorname{deg} \zeta .
$$

Since $h^{0}(E) \geqslant s+2$, by this lemma there must be an exact sequence

$$
0 \longrightarrow \xi(D) \longrightarrow E \longrightarrow \eta(-D) \rightarrow 0
$$

for some effective divisor $D$ of degree $d$ on $C$. Since $E$ is stable we must have

$$
\operatorname{deg}(\xi(D))=s+1+d \leqslant \operatorname{deg}(E) / 2=2 s,
$$

that is, $d \leqslant s-1$. But then, as in the proof of Lemma 5.3, we deduce that $d \leqslant 1$. Two cases can occur: either

$$
0 \longrightarrow \xi(p) \longrightarrow E \longrightarrow \eta(-p) \longrightarrow 0
$$

or

$$
0 \longrightarrow \xi \longrightarrow E \longrightarrow \eta \longrightarrow 0 \text {. }
$$

Then one concludes exactly as in [Muk96, pp. 195-196] by using Lemma 5.10 as follows. In the first case, $E \cong E_{p}$ because the extension does not split and is unique. In the second case, the 


\section{MUKAI PROGRAM}

coboundary

$$
H^{0}(C, \eta) \longrightarrow H^{1}(C, \xi)
$$

has rank one. We then apply point (ii) in Lemma 5.4 together with the fact that, by (4.2), the quadratic hull of $\Phi_{|\eta|}(C)$ is exactly $S$. We thus find a point $x \in S$ such that $H^{0}\left(S, I_{x} A\right)=$ $\operatorname{Im}\left[H^{0}(S, E) \longrightarrow H^{0}(C, \eta)=H^{0}(S, A)\right]$. By the uniqueness again we have $E=E_{x}=\mathcal{E}_{x_{\mid C}}$.

REMARK 5.11. The two vector bundles $\widetilde{E}_{L}$ and $E_{L}$ are also stable in the Rank-2 case, for every choice of a base-point-free pencil $|L|$ of degree $s+2$.

The proof of this fact runs as follows. By Theorem 5.1, it is enough to prove that $\widetilde{E}_{L}$ is stable. Suppose it is not, and let $N$ be a subsheaf of $\widetilde{E}_{L}$ with slope greater than or equal to $2 s=\mu_{C}\left(\widetilde{E}_{L}\right)$. Then $\alpha=N_{\mid C}$ destabilizes $E_{L}$. On the other hand, by Mukai's Lemma $5.10, \operatorname{Hom}\left(\xi, E_{L}\right) \neq 0$ and we have an exact sequence

$$
0 \longrightarrow \xi(D) \longrightarrow E_{L} \longrightarrow \eta(-D) \longrightarrow 0
$$

for some positive divisor $D$. We may write (5.13) with $E$ replaced by $E_{L}$ and conclude, in exactly the same way, that $\operatorname{deg}(D) \leqslant 1$. We can then proceed as in the proof of Lemma 5.3 and prove that either $\alpha=\eta$ or $\alpha=\eta(-p)$. On the other hand, we have an exact sequence

$$
0 \longrightarrow L \longrightarrow E_{L} \longrightarrow K_{C} L^{-1} \longrightarrow 0 \text {. }
$$

We must then have either $h^{0}\left(C, L \alpha^{-1}\right) \neq 0$ or $h^{0}\left(C, K_{C} L^{-1} \alpha^{-1}\right) \neq 0$. For degree reasons, the only possibility is that $\alpha=\eta(-p)$ and $h^{0}\left(C, K_{C} L^{-1} \alpha^{-1}\right) \neq 0$. This implies that $L=\xi(p)$, but then $L$ cannot be base-point free. This contradiction proves our claims.

Next, we prepare the ground for the proof of the last step. From the exact sequence (5.1), we deduce the following exact sequences:

$$
\begin{gathered}
0 \longrightarrow \mathcal{U} \longrightarrow S^{2} \mathcal{E}_{x} \longrightarrow \mathcal{O}_{S}\left(I_{x}^{2} A^{2}\right) \longrightarrow 0, \\
0 \longrightarrow \mathcal{O}_{S}(2 B) \longrightarrow \mathcal{U} \longrightarrow \mathcal{O}_{S}\left(I_{x}(A+B)\right) \longrightarrow 0 .
\end{gathered}
$$

In particular, we find that

$$
\mathcal{U} \cong \mathcal{E}_{x}(B)
$$

We also have

$$
\begin{gathered}
0 \longrightarrow U \longrightarrow S^{2} H^{0}\left(\mathcal{E}_{x}\right) \longrightarrow S^{2} H^{0}\left(I_{x} A\right) \longrightarrow 0, \\
0 \longrightarrow S^{2} H^{0}(S, B) \longrightarrow U \longrightarrow H^{0}(B) \otimes H^{0}\left(I_{x} A\right) \longrightarrow 0 .
\end{gathered}
$$

LEMMA 5.12. We have $H^{0}\left(S, S^{2} \mathcal{E}_{x}(-C)\right)=0$.

Proof. We have an exact sequence

$$
0 \longrightarrow\left(S^{2} \mathcal{E}_{x}\right)(-C) \longrightarrow S^{2} \mathcal{E}_{x} \longrightarrow S^{2} E_{x} \longrightarrow 0 .
$$

On the other hand, we have an exact sequence

$$
0 \longrightarrow \mathcal{E}_{x}(-A) \longrightarrow S^{2} \mathcal{E}_{x}(-C) \longrightarrow \mathcal{O}_{S}\left(I_{x}^{2}(A-B)\right) \longrightarrow 0 .
$$

Lemma 4.1 implies that $H^{0}\left(I_{x}^{2}(A-B)\right)=0$ and we see that

$$
H^{0}\left(\mathcal{E}_{x}(-A)\right)=0
$$




\section{E. Arbarello, A. Bruno and E. Sernesi}

by looking at the exact sequence

$$
0 \longrightarrow \mathcal{O}_{S}(B-A) \longrightarrow \mathcal{E}_{x}(-A) \rightarrow I_{x} \longrightarrow 0
$$

LEMma 5.13. (i) The map $S^{2} H^{0}\left(S, \mathcal{O}_{S}(B)\right) \rightarrow H^{0}\left(S, \mathcal{O}_{S}(2 B)\right)$ is an isomorphism.

(ii) The map $H^{0}\left(S, \mathcal{O}_{S}(B) \otimes H^{0}\left(S, I_{x}(A)\right) \rightarrow H^{0}\left(S, I_{x}(A+B)\right)\right.$ is injective.

(iii) The map $F: S^{2} H^{0}\left(S, I_{x}(A)\right) \rightarrow H^{0}\left(S, I_{x}^{2}(2 A)\right.$ is surjective.

Proof. (i) The first statement follows from the base-point-free-pencil trick.

(ii) The second statement again follows from the base-point-free-pencil trick.

(iii) Let $x \in S$. Let $A$ be a generic hyperplane section of $S$ given by the equation $s_{A}=0$ and assume $x \notin A$. Consider the following commutative diagram:

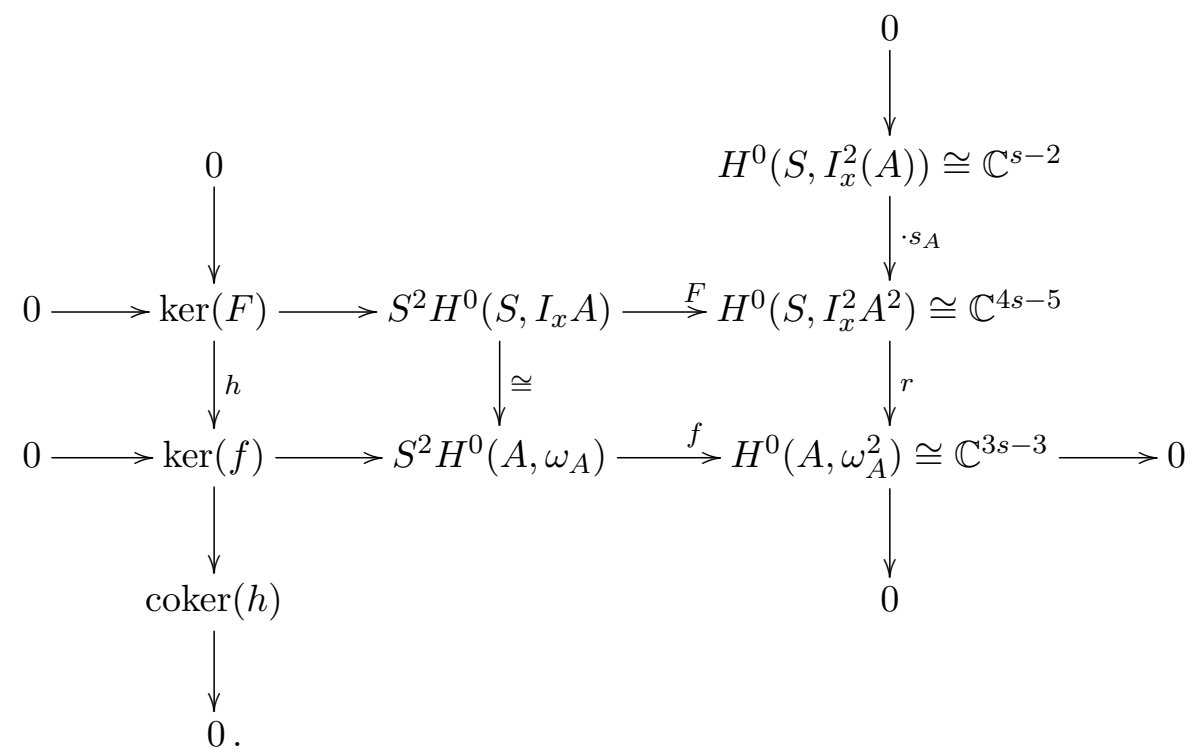

Consider an element $s_{A} \cdot t$ with $t \in H^{0}\left(S, I_{x}^{2} A\right)$. We can choose coordinates $\left\{x_{0}, \ldots, x_{s}\right\}$ such that

$$
x=[1,0, \ldots, 0], \quad s_{A}=x_{0}, \quad t=x_{1}, \quad T_{x}(S)=\left\{x_{1}=\cdots=x_{s-2}=0\right\} .
$$

To prove that $s_{A} \cdot t$ lies in $\operatorname{Im}(F)$, one must find $\widetilde{Q} \in S^{2} H^{0}\left(S, I_{x} A\right)$, that is, a quadric which is singular in $x$, such that $\widetilde{Q}_{\mid S}=\left(x_{0} x_{1}\right)_{\mid S}$. In other words, we must find a quadric $Q \in I_{S}(2)$ such that

is singular in $x$. We must have

$$
\widetilde{Q}=\lambda Q+\mu x_{0} x_{1}, \quad \mu \neq 0
$$

$$
0=\left(\frac{\partial \widetilde{Q}}{\partial x_{j}}\right)_{x}=\lambda\left(\frac{\partial Q}{\partial x_{j}}\right)_{x} \quad \text { for } \quad j \neq 1 \quad \text { and } \quad 0=\left(\frac{\partial \widetilde{Q}}{\partial x_{1}}\right)_{x}=\lambda\left(\frac{\partial Q}{\partial x_{1}}\right)_{x}+\mu
$$

Since the ideal of $S$ is generated by quadrics (Remark 4.2), we may choose $Q$ in $I_{S}(2)$ such that $T_{x}(Q)=\left\{x_{1}=0\right\}$, so that

$$
Q=x_{0} x_{1}+\sum_{i \neq 0, j \neq 0} b_{i j} x_{i} x_{j}
$$

We may then set $\widetilde{Q}=Q-x_{0} x_{1}$. 


\section{MUKAI PROGRAM}

We are now ready to prove the following result.

Proposition 5.14. For every $x \in S$,

$$
\operatorname{dim} T_{\left[E_{x}\right]}\left(M_{C}\left(2, K_{C}, s\right)\right)=2
$$

and the differential of $\sigma: M_{v}(S) \rightarrow M_{C}\left(2, K_{C}, s\right)$ is an isomorphism at $\left[\mathcal{E}_{x}\right]$.

Proof. In view of Proposition 3.3 we must verify the following three conditions:

(i) $H^{1}\left(S, S^{2} \mathcal{E}_{x}\right)=0$.

(ii) $H^{0}\left(S, S^{2} \mathcal{E}_{x}(-C)\right)=0$.

(iii) The map $S^{2} H^{0}\left(S, \mathcal{E}_{x}\right) \rightarrow H^{0}\left(S, S^{2} \mathcal{E}_{x}\right)$ is surjective.

We start with statement (iii). Look at (5.18), (5.20) and (5.21). We get a diagram

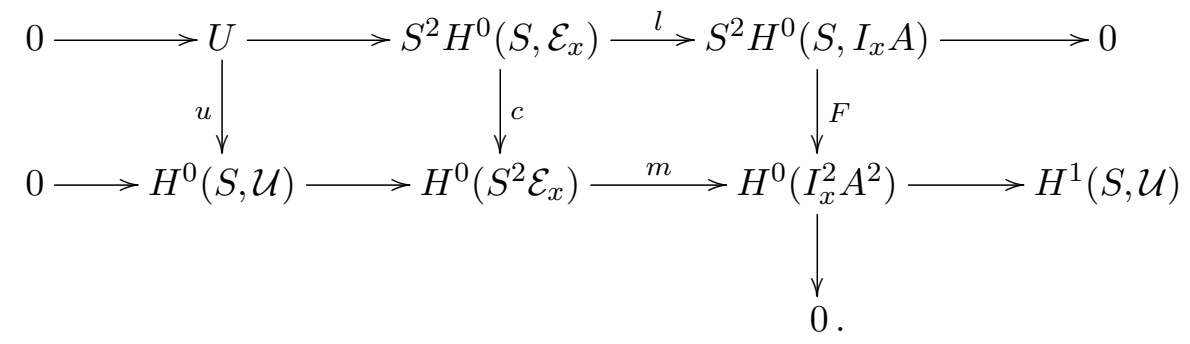

We will show that $u$ is an isomorphism. Let us first show that

$$
H^{1}(S, \mathcal{U})=0
$$

where $\mathcal{U}=\mathcal{E}_{x}(B)$. For this we look at the sequence

$$
0 \longrightarrow \mathcal{E}_{x} \longrightarrow \mathcal{E}_{x}(B) \longrightarrow \mathcal{E}_{x}(B)_{\mid B} \longrightarrow 0 \text {. }
$$

We get $H^{1}(\mathcal{U})=H^{1}\left(\mathcal{E}_{x}(B)\right)=H^{1}\left(\mathcal{E}_{x}(B)_{\mid B}\right)=H^{1}\left(\mathcal{E}_{x \mid B}\right)$. From the exact sequence

$$
0 \longrightarrow \mathcal{E}_{x}(-B) \longrightarrow \mathcal{E}_{x} \longrightarrow \mathcal{E}_{x \mid B} \longrightarrow 0
$$

we get

$$
H^{1}\left(\mathcal{E}_{x \mid B}\right) \cong H^{2}\left(\mathcal{E}_{x}(-B)\right) .
$$

However, $H^{2}\left(\mathcal{E}_{x}(-B)\right)=H^{0}\left(\mathcal{E}_{x}(-A)\right)=0$ by $(5.24)$. In conclusion, $H^{1}(\mathcal{U})=0$.

We now claim that $u$ is an isomorphism. Consider the diagram

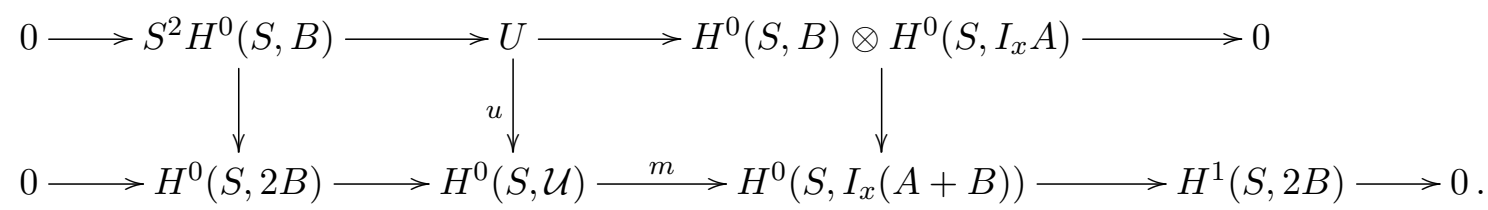

Since $S^{2} H^{0}(B) \rightarrow H^{0}(2 B)$ is an isomorphism and

$$
H^{0}(B) \otimes H^{0}\left(I_{x} A\right) \longrightarrow H^{0}\left(I_{x}(A+B)\right)
$$

is injective, the claim follows from a dimension count.

From Lemma 5.13, we know that $F$ is surjective and so is $F l$. Therefore $m$ is surjective and so is $c$, proving statement (iii).

Item (ii) is Lemma 5.12 . 


\section{E. Arbarello, A. Bruno and E. Sernesi}

To prove that condition (i) holds, we look at the exact sequence

$$
0 \longrightarrow H^{1}(\mathcal{U})=0 \longrightarrow H^{1}\left(S^{2} \mathcal{E}_{x}\right) \longrightarrow H^{1}\left(I_{x}^{2} A^{2}\right) \text {. }
$$

But now $H^{1}\left(I_{x}^{2} A^{2}\right)=0$, as follows from the exact sequences

$$
0 \longrightarrow I_{x} A^{2} \longrightarrow A^{2} \longrightarrow A_{\mid x}^{2} \longrightarrow 0, \quad 0 \longrightarrow I_{x}^{2} A^{2} \longrightarrow I_{x} A^{2} \longrightarrow A_{\mid x}^{2} \otimes I_{x} / I_{x}^{2} \longrightarrow 0
$$

and from the ampleness of $A$.

The proof of Theorem 5.1 is now complete.

\section{Brill-Noether loci in the Rank-1 case}

The purpose of this section is to prove the following result.

Theorem 6.1. Let $(S, C)$ be a general pair belonging to the Rank-1 case. There is a unique, generically smooth, two-dimensional irreducible component $V_{C}\left(2, K_{C}, s\right)$ of $M_{C}\left(2, K_{C}, s\right)$, containing the Voisin bundles $E_{L}$ with $L \in W_{s+2}^{1}(C)$, such that $\sigma$ induces an isomorphism of $M_{v}(S)$ onto $V_{C}\left(2, K_{C}, s\right)_{\text {red. }}$. In particular, $V_{C}\left(2, K_{C}, s\right)_{\text {red }}$ is a $K 3$ surface.

Before going into the proof we need some preliminaries. In the next statement we will refer to the notations introduced in diagram (1.1).

Lemma 6.2. Let $\left(S_{0}, C_{0}\right) \in \mathcal{P}_{g}$ be a pair belonging to the Rank-2 case. Then there exist a non-singular affine curve $B$ and a pair $(\mathcal{S}, \mathcal{C})$ with the following property. There is a diagram of smooth families over $B$

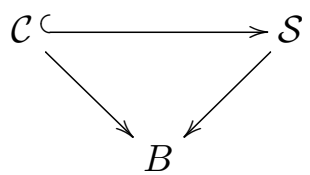

whose fibre $\left(\mathcal{S}\left(b_{0}\right), \mathcal{C}\left(b_{0}\right)\right)$ over $b_{0}$ is $\left(S_{0}, C_{0}\right)$, and such that for all $b \neq b_{0}$ outside a countable subset, the fibre $(\mathcal{S}(b), \mathcal{C}(b)) \in \mathcal{P}_{g}$ is a pair belonging to the Rank-1 case.

Proof. Let $\mathcal{H}_{g}$ be the open subset of the Hilbert scheme of $\mathbb{P}^{g}$ parametrizing non-singular K3 surfaces of degree $4 s$ and let $\mathcal{F}_{g} \rightarrow \mathcal{H}_{g}$ be the open subset of the flag Hilbert scheme parametrizing pairs $C \subset S \subset \mathbb{P}^{g}$ with $[S] \in \mathcal{H}_{g}$ and $C \in\left|\mathcal{O}_{S}(1)\right|$. Then $\left(S_{0}, C_{0}\right)$ corresponds to a point $b_{0} \in \mathcal{F}_{g}$. Let $B \subset \mathcal{F}_{g}$ be a general non-singular affine curve through $b_{0}$. Then the pullback to $B$ of the universal family over $\mathcal{F}_{g}$ has the required properties.

Proof of Theorem 6.1. The family (6.1) defines naturally a varying Mukai vector $v(b)$ such that $v=v\left(b_{0}\right) ;$ as $b \in B$ varies, the moduli spaces $M_{v(b)}(\mathcal{S}(b))$ fit into a family $\varphi: \mathcal{M}_{v}(\mathcal{S} / B) \rightarrow B$ of projective surfaces. Modulo shrinking $B$ if necessary, we may assume that this is a family of K3 surfaces. Similarly, the moduli spaces $M_{\mathcal{C}(b)}\left(2, K_{\mathcal{C}(b)}\right)$ fit into a smooth proper family $\mathcal{M}_{\mathcal{C} / B}\left(2, \omega_{\mathcal{C} / B}\right) \rightarrow B$ of relative dimension $3 g-3$. By the openness of (semi)stability ([Huy97, Proposition 2.3.1]) and the properness of $\varphi$, we may assume that the restriction morphisms

$$
\sigma_{b}^{\prime}: M_{v(b)}(\mathcal{S}(b)) \longrightarrow M_{\mathcal{C}(b)}\left(2, K_{\mathcal{C}(b)}\right)
$$




\section{MUKAI PROGRAM}

are well defined. They define a morphism of relative moduli spaces:

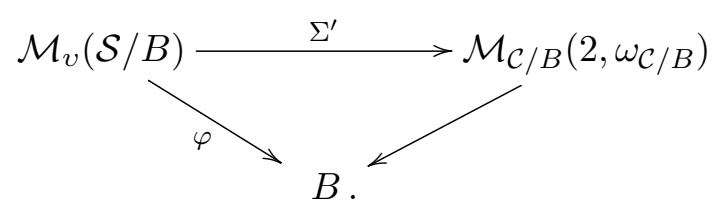

Over $b_{0}$ we have

$$
\Sigma^{\prime}\left(b_{0}\right)=\sigma_{0}^{\prime}: M_{v_{0}}\left(S_{0}\right) \longrightarrow M_{C_{0}}\left(2, K_{C_{0}}\right),
$$

which is an embedding, with image $M_{C_{0}}\left(2, K_{C_{0}}, s\right)=V_{C_{0}}\left(2, K_{C_{0}}, s\right)$. Therefore, modulo shrinking $B$ if necessary, we may assume that for all $b \in B$, we have that $\Sigma^{\prime}(b)=\sigma_{b}^{\prime}$ embeds the K3 surface $M_{v(b)}(\mathcal{S}(b))$ into $M_{\mathcal{C}(b)}\left(2, K_{\mathcal{C}(b)}\right)$, and the image is contained in $M_{\mathcal{C}(b)}\left(2, K_{\mathcal{C}(b)}, s\right)$.

Modulo performing an étale base change we may further assume that there is a line bundle $\mathcal{L}$ on $\mathcal{C}$ such that $\mathcal{L}(b) \in W_{s+2}^{1}(\mathcal{C}(b))$ and $L_{0}:=\mathcal{L}\left(b_{0}\right)$ is a base-point free $g_{s+2}^{1}$. Consider the corresponding family $\mathcal{E}_{\mathcal{L}}$ of Voisin bundles on $\mathcal{S}$. The vector bundle $\mathcal{E}_{\mathcal{L}}\left(b_{0}\right)$ over $S_{0}$ satisfies conditions (i),(ii) and (iii) of Proposition 3.3 as all bundles in $M_{v}\left(S_{0}\right)$ do (see Section 3). Therefore, by uppersemicontinuity, we may assume that all bundles $\mathcal{E}_{\mathcal{L}}(b)$ satisfy at least conditions (i) and (ii) as well. Moreover, by construction, they also satisfy $h^{0}\left(\mathcal{E}_{\mathcal{L}}(b)\right)=s+2$, so that, in particular, $S^{2} H^{0}\left(\mathcal{E}_{\mathcal{L}}(b)\right)$ has constant dimension. Moreover, they also satisfy $h^{0}\left(S^{2} \mathcal{E}_{\mathcal{L}}(b)\right)=h^{0}\left(S^{2} \mathcal{E}_{\mathcal{L}}(b)_{\mid \mathcal{C}(b)}\right)-2$, as shown by the exact sequence

$$
0 \longrightarrow S^{2} \mathcal{E}_{\mathcal{L}}(b)(-\mathcal{C}(b)) \longrightarrow S^{2} \mathcal{E}_{\mathcal{L}}(b) \longrightarrow S^{2} \mathcal{E}_{\mathcal{L}}(b)_{\mid \mathcal{C}(b)} \longrightarrow 0,
$$

because $h^{1}\left(S^{2} \mathcal{E}_{\mathcal{L}}(b)(-\mathcal{C}(b))\right)=2$. Therefore semicontinuity applies and condition (iii) can be also assumed to be satisfied for all $b \in B$.

We now apply Proposition 3.3 and deduce that $M_{\mathcal{C}(b)}\left(2, K_{\mathcal{C}(b)}, s\right)$ is smooth of dimension two at $\sigma_{b}\left(\mathcal{E}_{\mathcal{L}}(b)\right)$. Therefore $\sigma_{b}$ embeds $M_{v(b)}(\mathcal{S}(b))$ into an irreducible two-dimensional generically smooth component $V_{\mathcal{C}(b)}\left(2, K_{\mathcal{C}(b)}, s\right)$ of $M_{\mathcal{C}(b)}\left(2, K_{\mathcal{C}(b)}, s\right)$ whose reduction is therefore isomorphic to $M_{v(b)}(\mathcal{S}(b))$. This component is uniquely determined by the condition of containing the bundles $E_{\mathcal{L}(b)}$.

\section{The Fourier-Mukai transform}

As usual, we consider a pair $(S, C)$ which we assume to be either in the Rank-1 or in the Rank-2 case. If we are in the Rank- 1 case we denote by $T$ the $\mathrm{K} 3$ surface $V_{C}\left(2, K_{C}, s\right)_{\text {red }}$ introduced in the previous section. We do the same in the Rank-2 case where, by virtue of Theorem 5.1, we have $V_{C}\left(2, K_{C}, s\right)_{\text {red }}=M_{C}\left(2, K_{C}, s\right)$. In both cases we have an isomorphism

$$
\sigma: M_{v}(S) \stackrel{\cong}{\longrightarrow} T
$$

where $v=(2,[C], s)$. We will always view the K3 surface $T$ as a subvariety of $M_{C}\left(2, K_{C}\right)$. We further assume that

$$
s=2 t+1, \quad \text { that is, } g \equiv 3 \bmod 4 .
$$

Following Mukai's program ([Muk01, Remark 10.3]) and its implementation in genus eleven ([Muk96, Section 4]), we are going to prove the following theorem.

Theorem 7.1. There exists a Poincaré bundle $U \rightarrow C \times T$, unique up to isomorphism, having the following properties: Denote by $\pi_{C}: C \times T \rightarrow C$ and $\pi_{T}: C \times T \rightarrow T$ the two projections. 


\section{E. Arbarello, A. Bruno and E. Sernesi}

(i) We have $\mathcal{U}_{\mid C \times\{[E]\}} \cong E$ for all $[E] \in T \subset M_{C}\left(2, K_{C}\right)$.

(ii) We have $\operatorname{det}(\mathcal{U}) \cong K_{C} \otimes h_{\text {det }}$, where $h_{\text {det }}=\left(\operatorname{det} R^{1} \pi_{T *} \mathcal{U}\right) \otimes\left(\operatorname{det} \pi_{T *} \mathcal{U}\right)^{-1}$.

We also have the following properties.

(iii) The map $h_{\text {det }}$ is a polarization of genus $g$ on $T$.

(iv) For each $x \in C$, the vector bundle $\mathcal{U}_{x}=\mathcal{U}_{\mid\{x\} \times T}$ is stable and $\left[\mathcal{U}_{x}\right] \in M_{\widehat{v}}(T)$, where $\widehat{v}=\left(2, h_{\text {det }}, s\right)$.

(v) The morphism $C \rightarrow \widehat{T}=M_{\widehat{v}}(T)$ defined by $x \mapsto \mathcal{U}_{x}$ is an embedding.

(vi) The Fourier-Mukai transform $\left(\widehat{T}, \widehat{h}_{\text {det }}\right)$ of $\left(T, h_{\text {det }}\right)$ is isomorphic to $(S, h)$, where $h=[C]$.

Proof. Write

$$
M_{C}\left(2, K_{C}\right)=R / P G L(\nu), \quad R \subset \text { Quot },
$$

consider the quotient map

$$
p: R \longrightarrow M_{C}\left(2, K_{C}\right)
$$

and set $R^{\prime}=p^{-1}(T)$. Let $\widetilde{\mathcal{U}}$ be the restriction to $C \times R^{\prime}$ of the universal bundle over $C \times$ Quot. Consider the projection

$$
\pi_{R^{\prime}}: C \times R^{\prime} \longrightarrow R^{\prime}
$$

The sheaf $\pi_{R^{\prime} *} \widetilde{\mathcal{U}}$ is a vector bundle of rank $s+2$, while $\pi_{R^{\prime} *}\left(\widetilde{\mathcal{U}} \otimes K_{C}\right)$ is a vector bundle of rank $8 s$; indeed, $h^{0}\left(E \otimes K_{C}\right)=3 \operatorname{deg} K_{C}+2(1-g)=8 s$. Since $s=2 t+1$, the two integers $8 s$ and $s+2$ are relatively prime and we can find integers $x$ and $y$ such that $1+x(s+2)+y 8 s=0$.

Consider then the vector bundle

$$
\mathcal{V}=\tilde{\mathcal{U}} \otimes \pi_{R^{\prime}}^{*}\left(\operatorname{det}\left(\pi_{R^{\prime} *} \widetilde{\mathcal{U}}\right)^{x} \otimes \operatorname{det}\left(\pi_{R^{\prime} *}\left(\widetilde{\mathcal{U}} \otimes K_{C}\right)\right)^{y}\right)
$$

on $C \times R^{\prime}$. The action of a central element $c \in \mathbb{C}^{*} \subset G L(\nu)$ on the three factors is via multiplication by $1, c^{x(s+2)}$ and $c^{8 y s}$, respectively. Thus the vector bundle $\mathcal{V}$ is acted on by $\mathbb{P} G L(\nu)$ and descends to a Poincaré bundle $\mathcal{V}$ on $C \times T$. Since $T$ is regular, there exists a line bundle $L$ on $T$ such that

$$
\operatorname{det} \mathcal{V}=K_{C} \otimes L
$$

Thus

$$
\mathcal{V}^{\vee} \otimes \pi_{C}^{*} K_{C} \cong \mathcal{V} \otimes \pi_{T}^{*} L^{-1}
$$

As a consequence, by Serre duality, we have

$$
\left(R^{1} \pi_{T *} \mathcal{V}\right)^{\vee} \cong \pi_{T *}\left(\mathcal{V}^{\vee} \otimes \pi_{C}^{*} K_{C}\right) \cong \pi_{T *} \mathcal{V} \otimes L^{-1}
$$

It follows that

$$
h_{\text {det }}=\left(\operatorname{det} R^{1} \pi_{T *} \mathcal{V}\right) \otimes\left(\operatorname{det} \pi_{T *} \mathcal{V}\right)^{-1} \cong L^{s+2} \otimes\left(\operatorname{det} \pi_{T *} \mathcal{V}\right)^{-2} .
$$

Now the universal bundle

$$
\mathcal{U}=\mathcal{V} \otimes L^{t+1} \otimes\left(\operatorname{det} \pi_{T *} \mathcal{V}\right)^{-1}
$$

satisfies both properties (i) and (ii). The unicity follows from the fact that $\operatorname{Pic}(T)$ is torsion free.

Since properties (iii), (iv) and (v) are invariant under small deformations, we may limit ourselves to the Rank-2 case. In this case, we have the universal extension

$$
0 \longrightarrow \mathcal{O}_{S \times T}\left(\rho^{*} B\right) \longrightarrow \mathcal{F} \longrightarrow \mathcal{I}_{\Delta}\left(\rho^{*} A+\tau^{*}(B-A)\right) \longrightarrow 0
$$




\section{MUKAI PROGRAM}

where $\rho$ and $\tau$ are the projections $S \times T \rightarrow S$ and $S \times T \rightarrow T$, respectively. Moreover, we identify $S$ and $T$ via the isomorphism

$$
S \longrightarrow M_{v}(S) \stackrel{\sigma}{\longrightarrow} T, \quad x \longmapsto \mathcal{E}_{x} \longmapsto E_{x}=\mathcal{E}_{x \mid C}
$$

(cf. Theorem 5.1 and Proposition 5.7). We have

$$
\operatorname{det}\left(\mathcal{F}_{\mid C \times T}\right)=K_{C} \otimes \mathcal{O}(B-A) .
$$

Consider

$$
0 \longrightarrow \tau_{*} \mathcal{O}_{S \times T}\left(\rho^{*} B\right) \longrightarrow \tau_{*} \mathcal{F} \longrightarrow \tau_{*} \mathcal{I}_{\Delta}\left(\rho^{*} A+\tau^{*}(B-A)\right) \longrightarrow R^{1} \tau_{*} \mathcal{O}_{S \times T}\left(\rho^{*} B\right) \longrightarrow 0,
$$

which gives

$$
0 \longrightarrow H^{0}(\xi) \otimes \mathcal{O}_{T} \longrightarrow \tau_{*}\left(\mathcal{F}_{C \times T}\right) \longrightarrow H^{0}(\eta) \otimes \mathcal{O}_{T}(B-A) \longrightarrow \mathcal{O}_{T}(B) \longrightarrow 0 .
$$

We know that

$$
h_{\mathrm{det}}=L^{s+2} \otimes \operatorname{det} \tau_{*}\left(\mathcal{F}_{C \times T}\right)^{-2} .
$$

We then have

$$
L=\mathcal{O}(B-A), \quad \operatorname{det} \tau_{*}\left(\mathcal{F}_{C \times T}\right)=\mathcal{O}(s B-(s+1) A), \quad h_{\operatorname{det}}=\mathcal{O}(s A-(s-2) B) .
$$

Thus $h_{\text {det }}$ is a positive polarization and its genus is given by

$$
g\left(h_{\mathrm{det}}\right)=\frac{1}{2}(s A-(s-2) B)^{2}+1=2 s+1,
$$

proving property (iii).

In the Rank-2 case, the normalized Poincaré bundle is given by

$$
\mathcal{U}=\mathcal{F}_{\mid C \times T} \otimes \tau^{*} \mathcal{O}((t+1) A-t B) .
$$

Let $C^{\prime}$ be a smooth element in $|s A-(s-2) B|$. Set $B^{\prime}=(t+1) A-t B$ and $A^{\prime}=t A-(t-1) B$. Under the identification given by (7.2) we consider $C^{\prime}, A^{\prime}$ and $B^{\prime}$ as divisors in $T$. We may then consider the Rank-2 case given by the decomposition

$$
\operatorname{Pic}(T)=\mathbb{Z} \cdot A^{\prime} \oplus \mathbb{Z} \cdot B^{\prime}, \quad\left|C^{\prime}\right|=\left|A^{\prime}+B^{\prime}\right| .
$$

For this case the universal extension can be given by tensoring (7.1) by $\tau^{*} \mathcal{O}\left(B^{\prime}\right)$. For each $x \in S$, setting $\mathcal{U}_{x}=\mathcal{U}_{\mid\{x\} \times T}$, we get

$$
0 \longrightarrow \mathcal{O}_{T}\left(B^{\prime}\right) \longrightarrow \mathcal{U}_{x} \longrightarrow I_{x}\left(A^{\prime}\right) \longrightarrow 0 .
$$

We also get an isomorphism (Theorem 5.1)

$$
T \longrightarrow M_{\widehat{v}}(T), \quad x \longmapsto \mathcal{U}_{x}
$$

and a fortiori an embedding

$$
C \longrightarrow M_{\widehat{v}}(T), \quad x \longmapsto \mathcal{U}_{x}
$$

Finally, we want to show that $\left(\widehat{T}, \widehat{h}_{d e t}\right)=\left(M_{\widehat{v}}(T), \widehat{h}_{\text {det }}\right)$ may be identified with $(S, h)$. Since we have the isomorphism $\sigma: \widehat{S}=M_{v}(S) \rightarrow T$, we have $\left(\widehat{T}, \widehat{h}_{d e t}\right)=\left(\widehat{\widehat{S}}, h^{\prime}\right)=\left(S, h^{\prime}\right)$ for some polarization $h^{\prime}$ of genus $g$. In the Rank-1 case we necessarily have $h^{\prime}=h$. Let us show that in the Rank- 2 case we also have $\widehat{h}_{\text {det }}=h$. To simplify the notation we will prove the equivalent statement that $\widehat{h}=h_{\text {det }}$, which means that

$$
\widehat{[C]}=\left[C^{\prime}\right]=[s A-(s-2) B] .
$$




\section{E. Arbarello, A. Bruno and E. Sernesi}

From [Muk99], we recall the procedure one has to follow to construct $\widehat{h}$, starting from $h$.

We let $\widehat{S}=M_{v}(S)$, where $v=(2, h, s)$. Then $\widehat{S}$ is again a K3 surface and there is a universal family $\mathcal{F}$ on $\widehat{S} \times S$. Let

$$
c_{1}(\mathcal{F})=h+\phi \in H^{2}(S) \oplus H^{2}(\widehat{S}) \quad \text { and } \quad c_{2}^{m i d}(\mathcal{F}) \in H^{2}(S) \oplus H^{2}(\widehat{S})
$$

be the first Chern class of $\mathcal{E}$ and the middle Künneth component of the second Chern class, respectively. Define a class $\psi \in H^{2}(\widehat{S})$ by setting

$$
h \cup c_{2}^{\text {mid }}(\mathcal{F})=p \otimes \psi \in H^{4}(S) \oplus H^{2}(\widehat{S}),
$$

where $p$ is the fundamental class of $S$. Both $\phi$ and $\psi$ are algebraic by the Lefschetz theorem. Then the class $\widehat{h}$ is given by $\widehat{h}=\psi-2 s \phi$.

We now consider the Rank-2 case in which $\operatorname{Pic}(S) \cong \mathbb{Z} \cdot A \oplus \mathbb{Z} \cdot B$ and we look at exact sequence (7.1):

$$
0 \longrightarrow \mathcal{O}_{S \times \widehat{S}}\left(p^{*} B\right) \longrightarrow \mathcal{F} \longrightarrow I_{\Delta}\left(p^{*} A+q^{*}(B-A)\right) \longrightarrow 0
$$

We get

$$
c_{1}(\mathcal{F})=c_{1}\left(p^{*}(A+B)\right)+c_{1}\left(q^{*}(B-A)\right) .
$$

It follows that

$$
h=c_{1}\left(p^{*}(A+B)\right), \quad \phi=c_{1}\left(q^{*}(B-A)\right) .
$$

On the other hand, we have

$$
c_{2}(\mathcal{F})=c_{1}\left(p^{*}(B)\right) \cup\left(c _ { 1 } \left(p^{*}(A)+c_{1}\left(q^{*}(B-A)\right)+\Delta,\right.\right.
$$

so that

$$
c_{2}(\mathcal{F})^{\text {mid }}=c_{1}\left(p^{*}(B)\right) \cup\left(c_{1}\left(q^{*}(B-A)\right)+\Delta .\right.
$$

Therefore, as a class in $H^{2}(\widehat{S})$, the class $\psi$ satisfies

$$
\psi=((A+B \cdot B)[B-A]+[A+B]=(s+2) B-s A .
$$

We conclude that $\widehat{h}=s A-(s-2) B$.

\section{ACKNOWLEDGEMENTS}

We heartily thank Claire Voisin for suggesting a correction and for a number of very useful remarks. We would also like to thank Vittoria Bussi, Claudio Fontanari and Giulia Saccà for interesting conversations on the subject of this paper. We are grateful to the referee for making a number of very useful remarks that have contributed to improving the paper significantly.

\section{REFERENCES}

AC81 E. Arbarello and M. Cornalba, Footnotes to a paper of Beniamino Segre: "On the moduli of polygonal curves and on a complement to the Riemann existence theorem" (Italian) [Math. Ann. 100 (1928), 537-551; Jbuch 54,685], The number of $g_{d}^{1}$ 's on a general d-gonal curve, and the unirationality of the Hurwitz spaces of 4-gonal and 5-gonal curves, Math. Ann. 256 (1981), 341362. http://dx.doi.org/10.1007/BF01679702

AFO13 M. Aprodu, G. Farkas and A. Ortega, Minimal resolutions, Chow forms of K3 surfaces and Ulrich bundles, arXiv:1212.6248 (2013). 


\section{MUKAI PROGRAM}

BFT10 E. Ballico, C. Fontanari and L. Tasin, Singular curves on K3 surfaces, Sarajevo J. Math. 6(19) (2010), 165-168.

CLM93 C. Ciliberto, A. Lopez and R. Miranda, Projective degenerations of K3 surfaces, Gaussian maps, and Fano threefolds, Invent. Math. 114 (1993), 641-667. http://dx.doi.org/10.1007/ BF01232682

CP95 C. Ciliberto and G. Pareschi, Pencils of minimal degree on curves on a K3 surface, J. Reine Angew. Math. 460 (1995), 15-36.

GL87 M. Green and R. Lazarsfeld, Special divisors on curves on a K3 surface, Invent. Math. 89 (1987), 357-370. http://dx.doi.org/10.1007/BF01389083

Huy97 D. Huybrechts and M. Lehn, The geometry of moduli spaces of sheaves, Aspects Math., E31, Friedr. Vieweg \& Sohn, Braunschweig, 1997.

Knu02 A. L. Knutsen, Smooth curves on projective K3 surfaces, Math. Scand. 90 (2002), 215-231.

Lel13 M. Lelli-Chiesa, Stability of rank-3 Lazarsfeld-Mukai bundles on K3 surfaces, Proc. London Math. Soc. 107 (2) (2013), 451-479. http://dx.doi.org/10.1112/plms/pds087

Muk84 S. Mukai, Symplectic structure of the moduli space of sheaves on an abelian or K3 surface, Invent. Math. 77 (1984), 101-116.

Muk96 S. Mukai, Curves and K3 surfaces of genus eleven, in Moduli of vector bundles (Sanda, 1994; Kyoto, 1994), Lecture Notes in Math., vol. 179 (Dekker, New York, 1996), 189-197.

Muk99 S. Mukai, Duality of polarized K3 surfaces, in New trends in algebraic geometry (Warwick, 1996), London Math. Soc. Lecture Note Ser., vol. 264 (Cambridge Univ. Press, Cambridge, 1999), 311326. http://dx.doi.org/10.1017/CB09780511721540.012.

Muk01 S. Mukai, Non-abelian Brill-Noether theory and Fano 3-folds [translation of Sūgaku 49 (1997), no. 1, 1-24], Sugaku Expositions 14 (2001), 125-153.

Sai74 B. Saint-Donat, Projective models of K3 surfaces, Amer. J. Math. 96 (1974), 602-639.

Voi92 C. Voisin, Sur l'application de Wahl des courbes satisfaisant la condition de Brill-Noether-Petri, Acta Math. 168 (1992), 249-272. http://dx.doi.org/10.1007/BF02392980

Wah97 J. Wahl, On cohomology of the square of an ideal sheaf, J. Algebraic Geom. 6 (1997), 481-511.

Wah02 J. Wahl, Hyperplane sections of Calabi-Yau varieties, J. Reine Angew. Math. 544 (2002), 39-59. http://dx.doi.org/10.1515/crll.2002.027

E. Arbarello ea@mat.uniroma1.it

Dipartimento di Matematica, Sapienza, Università di Roma, Roma, Italy

A. Bruno bruno@mat.uniroma3.it

Dipartimento di Matematica e Fisica, Università Roma Tre, Roma, Italy

E. Sernesi sernesi@mat.uniroma3.it

Dipartimento di Matematica e Fisica, Università Roma Tre, Roma, Italy 